TITLE:

\title{
Geometry and topology optimization of plane frames for compliance minimization using force density method for geometry model
}

\author{
$\operatorname{AUTHOR(S):~}$
}

Shen, Wei; Ohsaki, Makoto

\section{CITATION:}

Shen, Wei ... [et al]. Geometry and topology optimization of plane frames for compliance minimization using force density method for geometry model. Engineering with

Computers 2021, 37: 2029-2046

\section{ISSUE DATE:}

2021-07

URL:

http://hdl.handle.net/2433/264257

\section{RIGHT:}

This is a post-peer-review, pre-copyedit version of an article published in En gineering with Computers. The final authenticated version is available online at: https://doi.org/10.1007/s00366-019-00923-w.; The full-text file will be made open to the public on $8 \mathrm{~J}$ anuary 2021 in accordance with publisher's 'Terms and Conditions for Self-Archiving'.; This is not the published version. Please cite only the published version. この論文は出版社版でありません。引用の際には出 版社版をご確認ざ利用ください。 


\title{
Geometry and Topology Optimization of Plane Frames for Compliance Minimization Using Force Density Method for Geometry Model
}

\author{
Wei Shen ${ }^{1 *}$ and Makoto Ohsaki ${ }^{1}$ \\ ${ }^{1}$ Dept. of Architecture and Architectural Engineering, Kyoto University, \\ Kyoto-Daigaku Katsura, Nishikyo, Kyoto 615-8540, Japan \\ * Corresponding author: shen.wei.28a@st.kyoto-u.ac.jp
}

\begin{abstract}
A new method is proposed for simultaneous optimization of shape, topology and crosssection of plane frames. Compliance against specified loads is minimized under constraint on structural volume. Difficulties caused by the melting nodes can be alleviated to some extent by introducing force density as design variables for defining the geometry, where the side constraints are assigned for force density to indirectly avoid the existence of extremely short members. Force density method is applied to an auxiliary cable net model with different boundary and loading conditions so that the regularity of force density matrix is ensured by positive force densities. Sensitivity coefficients of the objective and constraint functions with respect to the design variables are also explicitly calculated. After the optimal geometry of the frame is obtained, the topology is further improved by removing the thin members and combining closely spaced nodes. It is demonstrated in the numerical examples of three types of frames that rational geometry and topology can be achieved using the proposed method, and the effect of bending moment on the optimal solution is also discussed.
\end{abstract}

Keywords: Shape and topology optimization; force density method; sequential quadratic programming; compliance minimization; plane frame.

\section{Introduction}

Geometry and topology optimization of truss-like structures is one of the standard topics in structural optimization, and has received considerable attention since the pioneering work by Maxwell [1] and Michell [2]. Valuable contribution has been made in the past decades to the development of mathematical formulations and optimization methods in this field; see the review articles $[3,4]$ and text books $[5,6]$ for a comprehensive survey of optimization of truss-like structures, and the recent development of optimization of truss-like continua is summarized in Ref. [7].

Generally, optimization of truss-like structures can be classified into three categories; namely, sizing, geometry (or shape) and topology optimization. Among them, sizing optimization, which aims at finding the optimal cross-sectional properties of members, is commonly included in geometry and topology optimization, and the change of structural topology can be achieved in the standard framework of sizing optimization problem [8,9]. Based on the well-established ground structure method [10], the optimal topology of a truss-like structure is obtained by removing the unnecessary members from the set of potential connections of nodes with fixed locations and retaining the members with positive cross-sectional properties. However, since the nodal locations are unable to vary during the optimization process, it is necessary to work with as many nodes and 
members as possible, resulting in large number of design variables. On the other hand, if the locations of nodes are also considered as design variables in the optimization problem, one can start from a sparse ground structure instead, because the adjustment of nodal locations can usually lead to effective improvement of objective function value [11-13].

When geometry optimization is involved, however, one of the main difficulties is the existence of melting nodes, or coalescent nodes, resulting in extremely short member in the structure and making the stiffness matrix singular [14-16]. Moreover, the derivative of the objective function with respect to the nodal coordinates becomes discontinuous due to existence of extremely short members. The nodes should be allowed to move in a wide range of the design space to obtain the global optimal solution; however, undesirable intersection and overlapping of members may occur in the search process.

Czarnecki [17] solved a shape and topology optimization problem of truss structure, where the equilibrium equation is transformed such that the sensitivity analysis can be more easily and efficiently implemented in geometry linear and nonlinear condition. Sokol [18] transformed the truss optimization problem under compliance constraint into an equivalent formulation in which axial forces in members are the only state variables. However, since the stiffness matrix of rigidly jointed frame structure is different from that of pin-jointed truss structure, such transformation cannot be applied to the compliance minimization problem of frame structure.

Force density method (FDM) is primarily applied in the form-finding process of cable nets and tensegrity structures, where the nonlinear equilibrium equations with respect to the unknown nodal coordinates is converted to a set of linear equations by prescribing the force density which is defined as the ratio of member force to its length [19, 20]. Recently, Ohsaki and Hayashi [21] explored the merit of FDM to shape and topology optimization of pin-jointed trusses, in which the objective and constraint functions are expressed explicitly by force density only; thus, the problem caused by melting nodes is alleviated. Kimura et al. [22] optimized the shape of shear wall consisting of latticed block where force density is used as an auxiliary parameter for arrangement of lattice element. Descamps and Coelho [23] used force density as an intermediate variable in the formulation of compliance minimization problem. However, since shear force and bending moment exist in frames, the FDM for cable nets and trusses cannot be directly applied to frames.

In this paper, a new method is presented for geometry and topology optimization of plane frame structures, in which the FDM is used to prevent the generation of extremely short members. The unique feature of the proposed method is that the FDM is utilized only for generating feasible shape and topology; thus, the loading and boundary conditions of the truss or cable net for applying FDM are not related to the true conditions for the frame to be optimized. Therefore, the variables of the optimization problem are the force densities of the members of the auxiliary pin-jointed structure, and the parameters defining the cross-sectional properties of the frame members. It is shown in the numerical examples that enforcing small positive lower bound for force density enhances convergence property to obtain an optimal frame without melting nodes and intersecting members.

The paper is organized as follows: Section 2 introduces some notations of finite element method, which are used in the optimization procedure, and the basic idea of FDM is briefly described in Section 3. Section 4 formulates the optimization problem of frame structure in terms of force density and cross-sectional properties, and the side constraints on member length are assigned in terms of the constraints on force density. Section 5 derives the sensitivity coefficients for objective and constraint functions so as to reduce computation efforts, and a further optimization is given in 
Section 6 to improve the optimization result. Three numerical examples are investigated in Section 7 to verify the effectiveness of the proposed method. Finally, some conclusions are drawn in Section 8 .

\section{Plane frame model}

Consider a plane frame structure discretized by Euler-Bernoulli beam element [24]. The global coordinates, node numbers and local displacement numbers of the $i$ th beam element are shown in Fig. 1, and each element has six degrees of freedom due to the consideration of bending deformation.

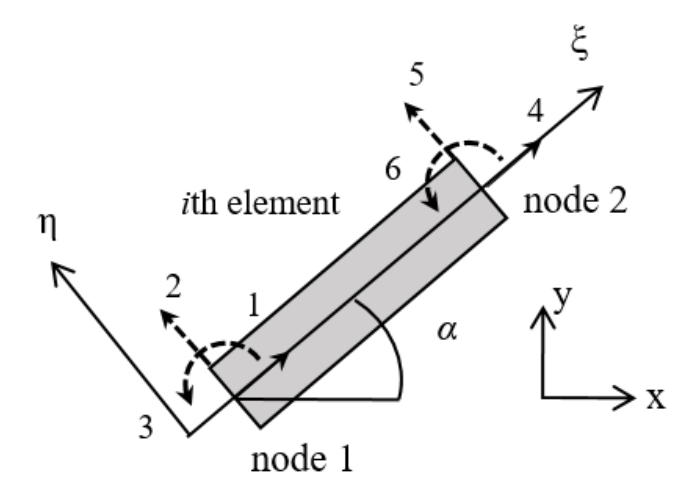

Fig. 1 Definition of displacement/force components and local coordinates of $i$ th beam element

Let $\alpha$ be the angle between the $\xi$-axis of the beam element and the $x$-axis of the global coordinates, where $\xi$-axis is directed from node 1 to node 2 . The local stiffness equation of $i$ th element can be written as

$$
\overline{\boldsymbol{k}}_{i} \overline{\boldsymbol{u}}_{i}=\overline{\boldsymbol{f}}_{i}
$$

where $\overline{\boldsymbol{k}}_{i} \in R^{6 \times 6}, \overline{\boldsymbol{u}}_{i} \in R^{6}$ and $\overline{\boldsymbol{f}}_{i} \in R^{6}$ are the stiffness matrix, nodal displacement vector and nodal force vector, respectively, with respect to the local coordinates. Note that the components in $\overline{\boldsymbol{f}}_{i}$ have the same order as those in $\overline{\boldsymbol{u}}_{i}$, which are specified in Fig. 1. By introducing transformation matrix $\boldsymbol{t}_{i} \in R^{6 \times 6}$ for the $i$ th element, the relation between the local stiffness matrix and the global stiffness matrix of $i$ th beam element can be obtained as

$$
\boldsymbol{k}_{i}=\boldsymbol{t}_{i}^{T} \overline{\boldsymbol{k}}_{i} \boldsymbol{t}_{i}
$$

where $\boldsymbol{k}_{i} \in R^{6 \times 6}$ represents the element stiffness matrix with respect to the global coordinates.

The vectors of nodal displacement and nodal force with respect to the global coordinates are denoted by $\boldsymbol{u}_{i} \in R^{6}$ and $\boldsymbol{f}_{i} \in R^{6}$, respectively. Relation between $\boldsymbol{u}_{i}$ and $\boldsymbol{f}_{i}$ can be expressed using $\boldsymbol{k}_{i}$ as

$$
\boldsymbol{k}_{i} \boldsymbol{u}_{i}=\boldsymbol{f}_{i}
$$

Assembling all the elements of $\boldsymbol{k}_{\boldsymbol{i}}$, we can derive the $s \times s$ overall stiffness matrix $\boldsymbol{K}$ of the frame 
structure, where $s$ is the number of degrees of freedom of the structure with appropriate boundary conditions. Accordingly, the nodal force vector $\boldsymbol{F} \in R^{s}$ and the nodal displacement vector $\boldsymbol{U} \in R^{s}$ can be constructed by assembling $\boldsymbol{f}_{i}$ and $\boldsymbol{u}_{i}$. Therefore, $\boldsymbol{U}$ is obtained by solving the following stiffness equation:

$$
\boldsymbol{K} \boldsymbol{U}=\boldsymbol{F}
$$

\section{Force density method}

FDM is widely used in the form-finding process of cable nets and tensegrity structures, where the force density is defined as the axial force divided by the length of a member. In this paper, the FDM is applied to an auxiliary pin-jointed structure to define the nodal locations of the frame that is to be optimized. Therefore, it is important to note again that the structure considered in this section is a pin-jointed truss or a cable net.

Let $m$ and $n$ represent the numbers of members and nodes of the auxiliary pin-jointed structure, respectively. If member $i$ connects nodes $j$ and $k(j \neq k)$, the $m \times n$ connectivity matrix $C$ can be constructed by defining each entry as [20]

$$
C_{(i, p)}=\left\{\begin{array}{cc}
1 & p=j \\
-1 & p=k \\
0 & \text { other case }
\end{array} \quad(i=1,2, \ldots, m ; j, k=1,2, \ldots, n)\right.
$$

Let $\boldsymbol{x}_{\text {free}}, \boldsymbol{y}_{\text {free }}$ and $\boldsymbol{x}_{\mathrm{fix}}, \boldsymbol{y}_{\mathrm{fix}}$ denote the $x$ - and $y$-coordinate vectors of free nodes and fixed nodes, respectively. The force density $q_{i}$ of the $i$ th member is defined as

$$
q_{i}=\frac{N_{i}}{L_{i}}
$$

where $N_{i}$ and $L_{i}$ are the axial force and the length of the $i$ th member, respectively. Accordingly, the force density vector is denoted by $\boldsymbol{q}=\left(q_{1}, q_{2}, \ldots q_{m}\right) \in R^{m}$, and the $m \times m$ force density matrix $\boldsymbol{Q}$ is given as $[21,25]$

$$
\boldsymbol{Q}=\boldsymbol{C}^{T} \operatorname{diag}(\boldsymbol{q}) \boldsymbol{C}
$$

where $\operatorname{diag}(\boldsymbol{q})$ represents the diagonal matrix that have the components of vector $\boldsymbol{q}$ in the diagonal terms. The columns of connectivity matrix $\boldsymbol{C}$ are arranged so that the columns corresponding to the free nodes precede those corresponding to the fixed nodes, i.e., $\boldsymbol{C}=\left(\boldsymbol{C}_{\mathrm{free}}, \boldsymbol{C}_{\mathrm{fix}}\right)$. Then, Eq. (7) can be rewritten as

$$
\boldsymbol{Q}=\left(\boldsymbol{C}_{\text {free }}, \boldsymbol{C}_{\text {fix }}\right)^{T} \operatorname{diag}(\boldsymbol{q})\left(\boldsymbol{C}_{\text {free }}, \boldsymbol{C}_{\text {fix }}\right)=\left[\begin{array}{ll}
\boldsymbol{C}_{\text {free }}^{T} \operatorname{diag}(\boldsymbol{q}) C_{\text {free }} & \boldsymbol{C}_{\text {free }}^{T} \operatorname{diag}(\boldsymbol{q}) \boldsymbol{C}_{\text {fix }} \\
\boldsymbol{C}_{\text {fix }}^{T} \operatorname{diag}(\boldsymbol{q}) C_{\text {free }} & \boldsymbol{C}_{\text {fix }}^{T} \operatorname{diag}(\boldsymbol{q}) \boldsymbol{C}_{\text {fix }}
\end{array}\right]
$$

and the equilibrium equations of free nodes and fixed nodes of a pin-jointed structure are written as follows [21]: 


$$
\begin{aligned}
& \boldsymbol{C}_{\text {free }}^{T} \operatorname{diag}(\boldsymbol{q}) \boldsymbol{C}_{\text {free }} \boldsymbol{x}_{\text {free }}+\boldsymbol{C}_{\text {free }}^{T} \operatorname{diag}(\boldsymbol{q}) \boldsymbol{C}_{\text {fix }} \boldsymbol{x}_{\text {fix }}=\boldsymbol{P}_{x, \text { free }} \\
& \boldsymbol{C}_{\text {free }}^{T} \operatorname{diag}(\boldsymbol{q}) \boldsymbol{C}_{\text {free }} \boldsymbol{y}_{\text {free }}+\boldsymbol{C}_{\text {free }}^{T} \operatorname{diag}(\boldsymbol{q}) \boldsymbol{C}_{\text {fix }} \boldsymbol{y}_{\text {fix }}=\boldsymbol{P}_{y, \text { free }} \\
& \boldsymbol{C}_{\text {fix }}^{T} \operatorname{diag}(\boldsymbol{q}) \boldsymbol{C}_{\text {free }} \boldsymbol{x}_{\text {free }}+\boldsymbol{C}_{\text {fix }}^{T} \operatorname{diag}(\boldsymbol{q}) \boldsymbol{C}_{\text {fix }} \boldsymbol{x}_{\text {fix }}=\boldsymbol{P}_{x, \text { fix }} \\
& \boldsymbol{C}_{\text {fix }}^{T} \operatorname{diag}(\boldsymbol{q}) \boldsymbol{C}_{\text {free }} \boldsymbol{y}_{\text {free }}+\boldsymbol{C}_{\text {fix }}^{T} \operatorname{diag}(\boldsymbol{q}) \boldsymbol{C}_{\text {fix }} \boldsymbol{y}_{\text {fix }}=\boldsymbol{P}_{y, \text { fix }}
\end{aligned}
$$

where $\boldsymbol{P}_{x, \text { free }}$ and $\boldsymbol{P}_{y}$,free are the vectors of external nodal loads applied at the free nodes in $x$ - and $y$ directions, respectively, and $\boldsymbol{P}_{x, \text { fix }}$ and $\boldsymbol{P}_{y}$,ix are the vectors of reaction forces at the fixed nodes. For a structure at self-equilibrium state, i.e., no external load applied at the free nodes, $\boldsymbol{P}_{x \text {,free }}$ and $\boldsymbol{P}_{y \text {,free }}$ are zero vectors.

Remark 1 For the conventional FDM, the free nodes and fixed nodes are determined in accordance with the support conditions. However, in this paper, the fixed nodes consist of the supported nodes and loaded nodes, as well as the nodes for specific reason, e.g. requirement of structural shape, that are not allowed to move during the optimization process

If the force densities are given for all members in the structure and the locations of fixed nodes are assigned, then the locations of free nodes can be obtained from Eq. (9), that is

$$
\begin{aligned}
& \boldsymbol{C}_{\text {free }}^{T} \operatorname{diag}(\boldsymbol{q}) \boldsymbol{C}_{\text {free }} \boldsymbol{x}_{\text {free }}=-\boldsymbol{C}_{\text {free }}^{T} \operatorname{diag}(\boldsymbol{q}) \boldsymbol{C}_{\text {fix }} \boldsymbol{x}_{\text {fix }}+\boldsymbol{P}_{x, \text { free }} \\
& \boldsymbol{C}_{\text {free }}^{T} \operatorname{diag}(\boldsymbol{q}) \boldsymbol{C}_{\text {free }} \boldsymbol{y}_{\text {free }}=-\boldsymbol{C}_{\text {free }}^{T} \operatorname{diag}(\boldsymbol{q}) \boldsymbol{C}_{\text {fix }} \boldsymbol{y}_{\text {fix }}+\boldsymbol{P}_{y, \text { free }}
\end{aligned}
$$

Therefore, $\boldsymbol{x}_{\text {free }}$ and $\boldsymbol{y}_{\text {free }}$ can be regarded as functions of $\boldsymbol{q}$.

Remark 2 It has been proved by Kanno and Ohsaki [26] that the submatrix corresponding to free nodes in the connectivity matrix, i.e., $\boldsymbol{C}_{\text {free, }}$ is non-singular if at least one node is fixed. Therefore, if $\boldsymbol{q}$ is a non-zero vector and location of at least one node is fixed, then the matrix $\boldsymbol{C}_{\text {free }}^{T} \operatorname{diag}(\boldsymbol{q}) \boldsymbol{C}_{\text {free }}$ is non-singular and Eq. (10) has a solution for $\boldsymbol{x}_{\text {free }}$ and $\boldsymbol{y}_{\text {free. }}$

\section{Optimization problem}

Consider an optimization problem of minimizing the compliance $F$, defined as follows:

$$
F=\boldsymbol{U}^{T} \boldsymbol{K} \boldsymbol{U}
$$

which is equal to the external work that is converted into the form of Eq. (11) using Eq. (4). Let $\boldsymbol{d}=\left(d_{1}, d_{2}, \ldots d_{m}\right)$ denote the vector of design variables defining the cross-sectional properties of beam members. The upper bound $\bar{V}$ is given for the total structural volume. Then, the conventional optimization problem with respect to the locations of free nodes and the cross-sectional properties as variables is formulated as follows:

$$
\begin{array}{lll}
\text { Minimize } & F\left(\boldsymbol{d}, \boldsymbol{x}_{\text {free }}, \boldsymbol{y}_{\text {free }}\right)=\boldsymbol{U}^{T} \boldsymbol{K}\left(\boldsymbol{d}, \boldsymbol{x}_{\text {free }}, \boldsymbol{y}_{\text {free }}\right) \boldsymbol{U} \\
\text { subject to } & d_{i, \text { lower }} \leq d_{i} \leq d_{i, \text { upper }}, \quad i=1,2, \ldots, m \\
& x_{j, \text { lower }} \leq x_{j} \leq x_{j, \text { upper }}, \quad y_{j, \text { lower }} \leq y_{j} \leq y_{j, \text { upper }}, \quad j=1,2, \ldots, n_{\text {free }} \\
& V\left(\boldsymbol{d}, \boldsymbol{x}_{\text {free }}, \boldsymbol{y}_{\text {free }}\right) \leq \bar{V}
\end{array}
$$


where $n_{\text {free }}$ is the number of free nodes, $d_{i, \text { lower }}$ and $d_{i \text {,upper }}$ are the lower and upper bounds for $d_{i}$, and $x_{j, \text { lower, }} x_{j \text {,upper, }} y_{j, \text { lower }}$ and $y_{j \text {,upper }}$ are the lower and upper bounds of $x$ - and $y$-coordinates of the $j$ th node. Note that $\boldsymbol{K}$ is a function of $\boldsymbol{d}, \boldsymbol{x}_{\text {free }}$ and $\boldsymbol{y}_{\text {free, }}$ while $\boldsymbol{U}$ is an implicit function of $\boldsymbol{d}, \boldsymbol{x}_{\text {free }}$ and $\boldsymbol{y}_{\text {free, }}$ which is obtained by solving Eq. (4). It should be noted here that since the stiffness matrix of rigidlyjointed frame structure differs from that of the pin-jointed truss structure, the compliance minimization problem (12) is not equivalent to the volume minimization under stress constraints, and cannot be solved by the existing methods for truss structure [17].

We next incorporate FDM for defining the nodal locations of the plane frame structure by solving Eq. (10) of the corresponding auxiliary truss or cable-net structure. The side constraints for the locations of free nodes are assigned in problem (12) to prevent divergence in the optimization process. However, they are no longer needed when the nodal coordinates can be calculated by Eq. (10). Accordingly, the optimization problem (12) is restated as

$$
\begin{array}{ll}
\text { Minimize } & F\left(\boldsymbol{d}, \boldsymbol{x}_{\text {free }}(\boldsymbol{q}), \boldsymbol{y}_{\text {free }}(\boldsymbol{q})\right)=\boldsymbol{U}^{T} \boldsymbol{K}\left(\boldsymbol{d}, \boldsymbol{x}_{\text {free }}(\boldsymbol{q}), \boldsymbol{y}_{\text {free }}(\boldsymbol{q})\right) \boldsymbol{U} \\
\text { Subject to } & d_{i, \text { lower }} \leq d_{i} \leq d_{i, \text { upper }}, \quad q_{i, \text { lower }} \leq q_{i} \leq q_{i, \text { upper }}, \quad i=1,2, \ldots, m \\
& V\left(\boldsymbol{d}, \boldsymbol{x}_{\text {free }}(\boldsymbol{q}), \boldsymbol{y}_{\text {free }}(\boldsymbol{q})\right) \leq \bar{V}
\end{array}
$$

where $q_{i, \text { lower }}$ and $q_{i, \text { upper }}$ are the lower and upper bounds for force density of the $i$ th member. We can observe from Eqs. (12) and (13) that these two formulations of optimization problem are basically the same, and they will lead to the same solution if a set of $\boldsymbol{q}$ in problem (13) can define the optimal solution of problem (12). In other words, if the optimal solution of problem (12) is included in the feasible domain of problem (13), it can be found by resolving the optimization problem (13). Recall that in Eq. (13) the structural compliance is calculated for the rigidly-jointed frame model with EulerBernoulli beam elements shown in Section 2.

Remark 3 One of the difficulties in solving problem (12) is the existence of melting or coalescent nodes, which will result in the singularities in $\boldsymbol{K}$ [14]. This can be handled by adding constraints to limit the lower bound of the member length. However, as pointed out by Achtziger [14], directly introducing such constraints to avoid geometries without melting nodes will in return cause intractable definition of feasible region of the nodal location. By contrast, the solution space becomes too small if a tight bound is given for the nodal coordinates, and large bound will lead to unfavorable intersection of members.

Substituting $q_{i}$ in Eq. (6) into the constraints on $q_{i}$ in problem (13), we obtain

$$
q_{i, \text { lower }} \leq \frac{N_{i}}{L_{i}} \leq q_{i, \text { upper }}
$$

If we assume the absolute value of $q_{i, \text { lower }}$ is equal to $q_{i \text {,upper, }}$, i.e., $\left|q_{i, \text { lower }}\right|=\left|q_{i, \text { upper }}\right|=q_{i \text {,abs }}(>0)$, Eq. (14) can be simplified as

$$
\left|\frac{N_{i}}{L_{i}}\right| \leq q_{i, \mathrm{abs}}
$$

Because the member lengths are positive, we can derive the lower-bound constraint for member 
length, if $N_{i}$ is not equal to 0 , by transforming Eq. (15) to

$$
\frac{\left|N_{i}\right|}{q_{i, \mathrm{abs}}} \leq L_{i}
$$

Since $q_{i}$ can have either positive or negative value, the nodal locations obtained from Eq. (10) will have drastic variation due to the change of sign of force density. Therefore, we next assume that

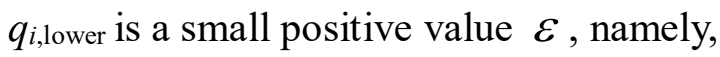

$$
q_{i, \text { lower }}=\varepsilon
$$

and $q_{i \text {,upper }}$ is a sufficiently large real number. Then Eq. (14) can be rewritten as

$$
\varepsilon \leq \frac{N_{i}}{L_{i}} \leq q_{i, \text { upper }}
$$

which means $q_{i}=N_{i} / L_{i}$ for all members are positive like a cable net during the optimization process. Since $N_{i}>0$ for all the members, we can reformulate Eq. (18) with respect to $L_{i}$ as

$$
\frac{N_{i}}{q_{i, \text { upper }}} \leq L_{i} \leq \frac{N_{i}}{\varepsilon}
$$

Hence, the length of $i$ th member is indirectly controlled by the side constraints of its corresponding force density. If $q_{i, \text { upper }}$ is appropriately chosen and the configuration of the frame is modeled by an auxiliary cable net that can be at self-equilibrium state with positive forces in all the members, , then the existence of extremely short member can be avoided by using Eq. (19).

Furthermore, for the auxiliary structure of cable net without external load, nodal locations are determined only by the ratios of force densities, and the nodal locations do not change when the force densities are scaled with the same factor because the external loads in Eq. (10) vanish. Therefore, to prevent poor convergence of optimization process due to non-uniqueness of the solution, an additional norm constraint with a constant $c$ is given for the optimization problem.

Hence, the optimization problem is rewritten as

$$
\begin{array}{ll}
\text { Minimize } & F\left(\boldsymbol{d}, \boldsymbol{x}_{\text {free }}(\boldsymbol{q}), \boldsymbol{y}_{\text {free }}(\boldsymbol{q})\right)=\boldsymbol{U}^{T} \boldsymbol{K}\left(\boldsymbol{d}, \boldsymbol{x}_{\text {free }}(\boldsymbol{q}), \boldsymbol{y}_{\text {free }}(\boldsymbol{q})\right) \boldsymbol{U} \\
\text { subject to } & d_{i, \text { lower }} \leq d_{i} \leq d_{i, \text { upper }}, \quad q_{i, \text { lower }} \leq q_{i} \leq q_{i, \text { upper }}, \quad i=1,2, \ldots, m \\
& V\left(\boldsymbol{d}, \boldsymbol{x}_{\text {free }}(\boldsymbol{q}), \boldsymbol{y}_{\text {free }}(\boldsymbol{q})\right) \leq \bar{V} \\
& \sum_{i=1}^{m}\left(q_{i}\right)^{2}=c
\end{array}
$$

in which $\boldsymbol{q}=\left(q_{1}, q_{2}, \ldots q_{m}\right)$ and $\boldsymbol{d}=\left(d_{1}, d_{2}, \ldots d_{m}\right)$ are the $2 m$ design variables, and the difficulties caused by the melting nodes are avoided by indirectly adding the side constraints on the force density vector, which in return control the upper and lower bounds of member length. 
Note that optimization problems (13) and (20) are classified as nonlinear programming (NLP) problem, in which all functions are differentiable with respect to $\boldsymbol{q}$ and $\boldsymbol{d}$. We will exploit this good nature to carry out the sensitivity analysis in the next section.

\section{Sensitivity Analysis}

To solve problems (13) and (20) using a gradient-based algorithm, sensitivity coefficients of objective and constraint functions are analytically derived for reducing computational effort [27, 28].

Differentiating the compliance with respect to $q_{i}$, we obtain

$$
\frac{\partial F}{\partial q_{i}}=\sum_{j=1}^{n_{\text {free }}}\left(\frac{\partial F}{\partial x_{\text {free }, j}} \frac{\partial x_{\text {free }, j}}{\partial q_{i}}+\frac{\partial F}{\partial y_{\text {free }, j}} \frac{\partial y_{\text {free }, j}}{\partial q_{i}}\right), \quad i=1,2, \ldots, m
$$

The differential coefficients $\partial F / \partial x_{\text {free, } j}$ and $\partial F / \partial y_{\text {free, } j}$ are called shape sensitivity coefficients, and the derivatives of the coordinates of free nodes $\boldsymbol{x}_{\text {free }}$ and $\boldsymbol{y}_{\text {free }}$ with respect to force density $\boldsymbol{q}$ can be computed from the equilibrium equation (10), and details can be found in Ref. [21]. Since there is only one response quantity, i.e. the compliance of the structure, the adjoint variable method is more effective than the direct differential method $[6,29]$ for computing the shape sensitivity coefficients.

The shape sensitivity coefficients $\partial F / \partial x_{\text {free, } j}$ and $\partial F / \partial y_{\text {free, } j}$ are expressed as follows:

$$
\frac{\partial F}{\partial x_{\text {free }, j}}=-\boldsymbol{U}^{T} \frac{\partial \boldsymbol{K}}{\partial x_{\text {free }, j}} \boldsymbol{U}, \quad \frac{\partial F}{\partial y_{\text {free }, j}}=-\boldsymbol{U}^{T} \frac{\partial \boldsymbol{K}}{\partial y_{\text {free }, j}} \boldsymbol{U}, \quad j=1,2, \ldots, n_{\text {free }}
$$

which are similar to the expression of design sensitivity coefficients with respect to $d_{i}$ as

$$
\frac{\partial F}{\partial d_{i}}=-\boldsymbol{U}^{T} \frac{\partial \boldsymbol{K}}{\partial d_{i}} \boldsymbol{U}, \quad i=1,2, \ldots, m
$$

Let $\left(x_{1, i}, y_{1, i}\right)$ and $\left(x_{2, i}, y_{2, i}\right)$ denote the coordinates of the two end nodes of $i$ th member. Differentiation of the total structural volume with respect to $q_{i}$ and $d_{i}$, respectively, leads to

$$
\frac{\partial V}{\partial q_{i}}=\sum_{j=1}^{m} \sum_{k=1}^{2} A_{j}\left(\frac{\partial L_{j}}{\partial x_{k, j}} \frac{\partial x_{k, j}}{\partial q_{i}}+\frac{\partial L_{j}}{\partial y_{k, j}} \frac{\partial y_{k, j}}{\partial q_{i}}\right), \quad \frac{\partial V}{\partial d_{i}}=L_{i} \frac{\partial A_{i}}{\partial d_{i}}, \quad i=1,2, \ldots, m
$$

Since the locations of fixed nodes are invariant to force density, the derivatives of member length $L_{i}$ with respect to $x_{k, i}$ and $y_{k, i}(k=1,2)$ vanish if $x_{k, i}$ and $y_{k, i}$, respectively, are the coordinates of a fixed node. The sensitivity coefficients of objective and constraint functions can be computed by using Eqs. (21)-(24).

\section{Improvement of optimal solution}

Since problems (13) and (20) may have many local optimal solutions, we solve this problem many times from randomly generated different initial solutions, and select the best local optimal solution as an optimal solution. However, the optimal solution may have closely spaced nodes and/or very thin members, causing ambiguity in structural layout. Therefore, we further optimize the crosssectional properties and nodal locations by solving the following problem: 


$$
\begin{array}{ll}
\text { Minimize } & F\left(\boldsymbol{d}, \boldsymbol{x}_{\text {free }}, \boldsymbol{y}_{\text {free }}\right)=\boldsymbol{U}^{T} \boldsymbol{K}\left(\boldsymbol{d}, \boldsymbol{x}_{\text {free }}, \boldsymbol{y}_{\text {free }}\right) \boldsymbol{U} \\
\text { subject to } & d_{i, \text { lower }} \leq d_{i} \leq d_{i, \text { upper }}, \quad i=1,2, \ldots, \hat{m} \\
& x_{j, \text { lower }} \leq x_{j} \leq x_{j, \text { upper }}, y_{j, \text { lower }} \leq y_{j} \leq y_{j, \text { upper }}, \quad j=1,2, \ldots, \hat{n}_{\text {free }} \\
& V\left(\boldsymbol{d}, \boldsymbol{x}_{\text {free }}, \boldsymbol{y}_{\text {free }}\right) \leq \bar{V}
\end{array}
$$

where $\hat{n}_{\text {free }}$ and $\hat{m}$ are the numbers of free nodes and members, respectively, after merging the closely spaced nodes of the optimal solution of problem (13) or (20). The same symbols in Section 4 are used for variables for brevity, and sensitivity analysis of the functions in problem (25) can be carried out in a similar manner as Section 5.

Note that in Section 4 the lower bound of member length is indirectly assigned in order to prevent the existence of zero-length member; however, the closely spaced nodes may still exist if the ratio

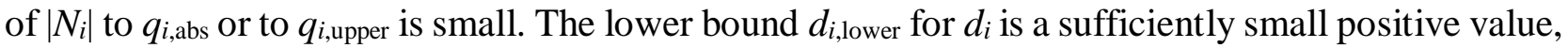
and owing to the fact that optimization problem of minimizing compliance is continuously dependent on the bounds of member volumes [30], optimal topology is further enhanced and obtained by removing some of the members whose member size reaches the lower bound $d_{i \text {,lower. }}$

\section{Numerical Examples}

In this section, we will present three numerical examples in order to illustrate the effectiveness of the proposed method. The nonlinear programming problem is solved using the sequential quadratic programming (SQP) algorithm in the fmincon of Optimization Toolbox of Matlab 2018a [31].

Each member is assumed to have solid circular cross-section, and thereby the cross-sectional area and second moment of inertia can be expressed by its diameter, i.e., the design variable $d_{i}$ is the diameter of member $i$. Without loss of generality, Young's modulus is assumed to be the same for all the members.

Optimal solutions are found for the following Cases $\mathrm{N}$ and $\mathrm{P}$ with negative and positive lower bounds, respectively, of force density:

Case N: Solve problem (13) with $-q_{i, \mathrm{abs}} \leq q_{i} \leq q_{i, \mathrm{abs}}, q_{i, \mathrm{abs}}=1000(\mathrm{~N} / \mathrm{m})$.

Case P: Solve problem (20) with $\varepsilon \leq q_{i} \leq q_{i, \mathrm{abs}}, q_{i, \mathrm{abs}}=1000(\mathrm{~N} / \mathrm{m}), \varepsilon=0.0001(\mathrm{~N} / \mathrm{m})$.

Note that the value of $q_{i, \text { abs }}$ is determined so that the lower bound of member length is around 0.001 $\mathrm{m}$ for a member with the axial force about $1 \mathrm{~N}$.

For the diameter, the lower bound is $0.001 \mathrm{~m}$, and no upper bound is given. We select the best result out of 100 solutions obtained from 100 random seeds.

\subsection{Example 1}

The first example is optimization of a two-dimensional cantilever frame. The initial ground structure consists of a $3 \times 2$ grid, with $n=12$ nodes and $m=27$ members, as shown in Fig. 2 . The frame is pinsupported at three left nodes $1,2,3$, and a downward vertical load $P$ with unit magnitude $1 \mathrm{~N}$ is applied at node 11. Note that the magnitude of load is not important, because the compliance is proportional to the load for the same optimal solution. According to Section 2, these four nodes, i.e., nodes 1, 2, 3 and 11 are the fixed nodes and the remaining eight nodes are the free nodes. 


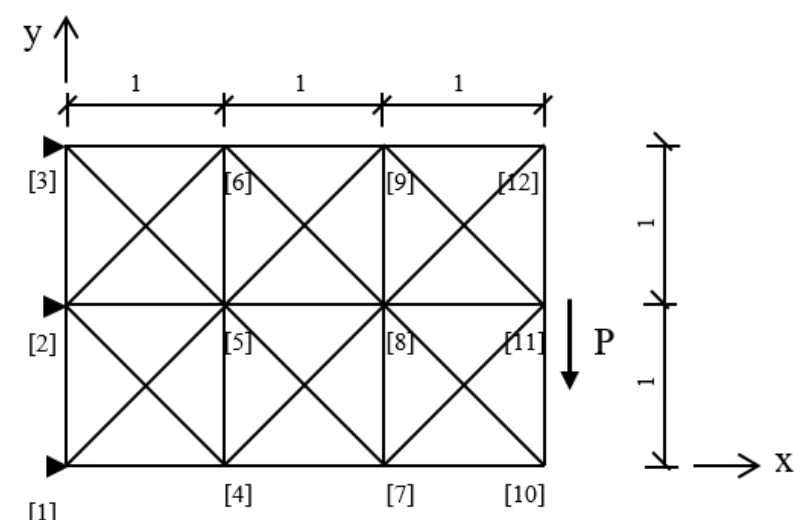

Fig. 2 Initial ground structure of Example 1

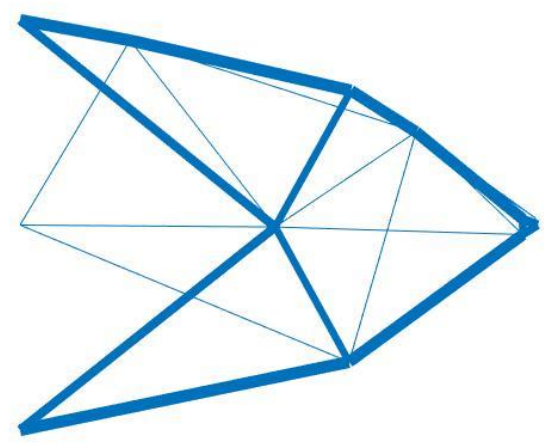

(a)

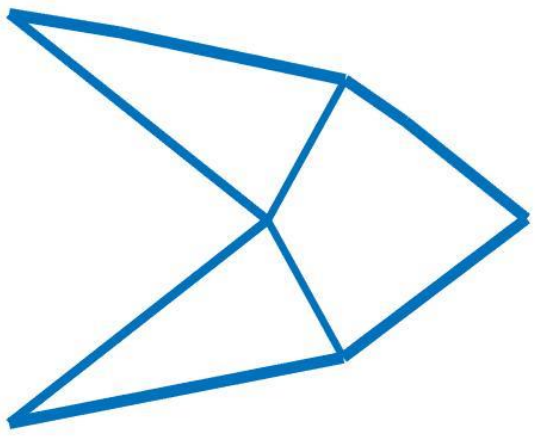

(b)

Fig. 3 Optimal shapes of Example 1 with Case $\mathrm{N}$ and $\bar{V}=1\left(\mathrm{~m}^{3}\right)$; (a) best result of problem (13), (b) optimal solution after solving problem (25)

Table 1. Nodal location of best result of Example 1 with $\bar{V}=1\left(\mathrm{~m}^{3}\right)$

\begin{tabular}{ccccc}
\hline \multirow{2}{*}{ Node } & \multicolumn{2}{c}{ Case N } & \multicolumn{2}{c}{ Case P } \\
\cline { 2 - 5 } & $x$ & $y$ & $x$ & $y$ \\
\hline 1 & 0 & 0 & 0 & 0 \\
2 & 0 & 1 & 0 & 1 \\
3 & 0 & 2 & 0 & 2 \\
4 & 1.9065 & 0.3324 & 0.3396 & 0.0567 \\
5 & 1.4783 & 0.9906 & 1.5071 & 1.0066 \\
6 & 0.6368 & 1.9009 & 0.6521 & 1.8924 \\
7 & 2.9136 & 0.9536 & 1.9760 & 0.3278 \\
8 & 2.2941 & 1.4542 & 1.5457 & 1.0069 \\
9 & 1.9173 & 1.6558 & 1.9596 & 1.6778 \\
10 & 2.9127 & 1.0291 & 3 & 0 \\
11 & 3 & 1 & 3 & 1 \\
12 & 2.9849 & 1.0351 & 3 & 2 \\
\hline
\end{tabular}

First consider Case $\mathrm{N}$ to solve problem (13) with the upper bound volume $\bar{V}$ equal to $1 \mathrm{~m}^{3}$. The best result among 100 trials is shown in Fig. 3(a) that has the compliance $F=81.957(\mathrm{Nm})$. Note that the width of each member in the following figures is proportional to its diameter, which is scaled 
appropriately for each figure. The locations of nodes are listed in Table 1, and the values of force density vector $\boldsymbol{q}$, member diameter vector $\boldsymbol{d}$ and member lengths $\boldsymbol{L}$ of the best result are listed in Table 2.

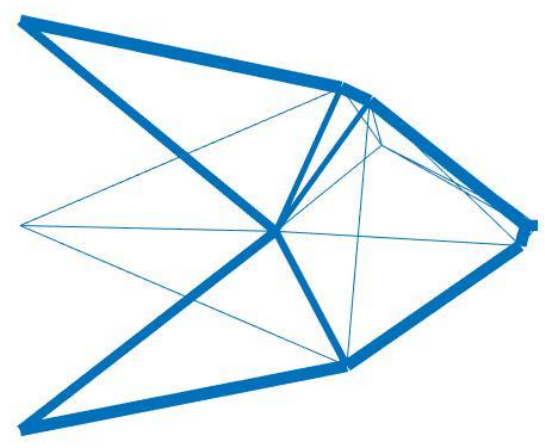

(a)

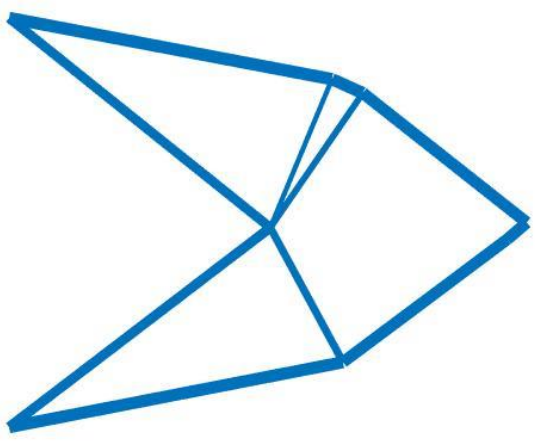

(b)

Fig. 4 Optimal shapes of Example 1 with Case $\mathrm{N}$ and $\bar{V}=0.7\left(\mathrm{~m}^{3}\right)$; (a) best result of problem (13), (b) optimal solution after solving problem (25)

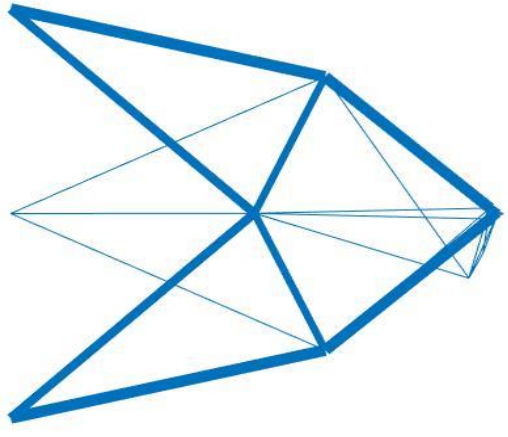

(a)

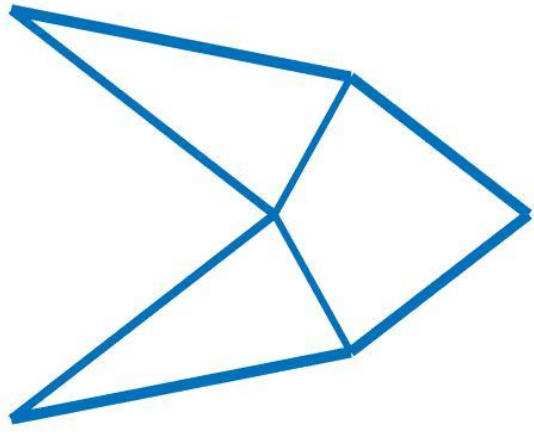

(b)

Fig. 5 Optimal shapes of Example 1 with Case $\mathrm{N}$ and $\bar{V}=0.4\left(\mathrm{~m}^{3}\right)$; (a) best result of problem (13), (b) optimal solution after solving problem (25)

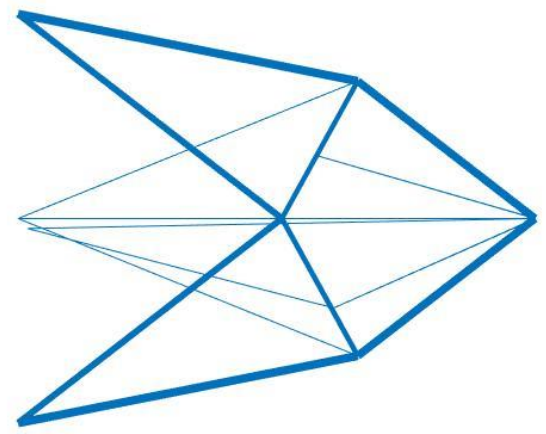

(a)

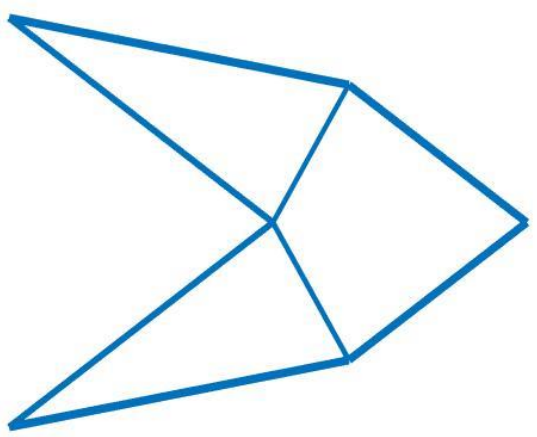

(b)

Fig. 6 Optimal shapes of Example 1 with Case $\mathrm{N}$ and $\bar{V}=0.1\left(\mathrm{~m}^{3}\right)$; (a) best result of problem (13), (b) optimal solution after solving problem (25) 
Table 2. Force density, diameter and length of best result of Example 1 with $\bar{V}=1\left(\mathrm{~m}^{3}\right)$

\begin{tabular}{|c|c|c|c|c|c|c|c|c|}
\hline \multirow{2}{*}{ Member } & \multirow{2}{*}{\multicolumn{2}{|c|}{ Nodes }} & \multicolumn{3}{|c|}{ Case $\mathrm{N}$} & \multicolumn{3}{|c|}{ Case P } \\
\hline & & & $q_{i}$ & $d_{i}$ & $L_{i}$ & $q_{i}$ & $d_{i}$ & $L_{i}$ \\
\hline 1 & 1 & 4 & -0.0953 & 0.379 & 1.935 & 0.632 & 0.374 & 0.344 \\
\hline 2 & 4 & 7 & 0.114 & 0.364 & 1.183 & 0.131 & 0.373 & 1.658 \\
\hline 3 & 7 & 10 & -0.124 & 0.243 & 0.075 & 0.283 & 0.001 & 1.075 \\
\hline 4 & 2 & 5 & -0.147 & 0.001 & 1.478 & 0.0001 & 0.001 & 1.507 \\
\hline 5 & 5 & 8 & 0.297 & 0.00399 & 0.938 & 0.192 & 0.186 & 0.038 \\
\hline 6 & 8 & 11 & -0.274 & 0.001 & 0.839 & 0.125 & 0.001 & 1.454 \\
\hline 7 & 3 & 6 & -0.380 & 0.380 & 0.644 & 0.456 & 0.371 & 0.661 \\
\hline 8 & 6 & 9 & 0.509 & 0.382 & 1.304 & 0.227 & 0.371 & 1.325 \\
\hline 9 & 9 & 12 & 0.325 & 0.00554 & 1.235 & 0.339 & 0.001 & 1.089 \\
\hline 10 & 4 & 5 & -0.220 & 0.232 & 0.785 & 0.0001 & 0.00108 & 1.505 \\
\hline 11 & 7 & 8 & 0.174 & 0.00163 & 0.796 & 0.00284 & 0.240 & 0.804 \\
\hline 12 & 10 & 11 & -0.300 & 0.295 & 0.092 & 0.0734 & 0.001 & 1 \\
\hline 13 & 5 & 6 & -0.237 & 0.001 & 1.240 & 0.0001 & 0.00193 & 1.231 \\
\hline 14 & 8 & 9 & -0.331 & 0.365 & 0.427 & 0.0001 & 0.238 & 0.788 \\
\hline 15 & 11 & 12 & -0.336 & 0.0352 & 0.038 & 0.0533 & 0.001 & 1 \\
\hline 16 & 1 & 5 & 0.0116 & 0.287 & 1.780 & 0.0148 & 0.295 & 1.812 \\
\hline 17 & 2 & 4 & 0.190 & 0.001 & 2.020 & 0.0001 & 0.001 & 1.002 \\
\hline 18 & 4 & 8 & -0.0752 & 0.001 & 1.187 & 0.0001 & 0.00308 & 1.535 \\
\hline 19 & 5 & 7 & -0.156 & 0.001 & 1.436 & 0.177 & 0.0121 & 0.825 \\
\hline 20 & 7 & 11 & -0.0187 & 0.255 & 0.098 & 0.00878 & 0.356 & 1.225 \\
\hline 21 & 8 & 10 & -0.0425 & 0.367 & 0.751 & 0.000574 & 0.001 & 1.768 \\
\hline 22 & 2 & 6 & 0.224 & 0.001 & 1.103 & 0.0001 & 0.001 & 1.105 \\
\hline 23 & 3 & 5 & 0.132 & 0.285 & 1.790 & 0.00141 & 0.296 & 1.805 \\
\hline 24 & 5 & 9 & -0.291 & 0.234 & 0.797 & 0.201 & 0.00782 & 0.809 \\
\hline 25 & 6 & 8 & -0.333 & 0.001 & 1.716 & 0.0001 & 0.00492 & 1.258 \\
\hline 26 & 8 & 12 & -0.509 & 0.0109 & 0.808 & 0.00253 & 0.001 & 1.761 \\
\hline 27 & 9 & 11 & 0.280 & 0.001 & 1.266 & 0.0343 & 0.355 & 1.241 \\
\hline
\end{tabular}

Table 3. Statistical result, final values of compliance $F(\mathrm{Nm})$ and CPU time (sec) of 100 results of Example 1 with different $\bar{V}\left(\mathrm{~m}^{3}\right)$

\begin{tabular}{ccccccccc}
\hline $\bar{V}$ & \multicolumn{2}{c}{1} & \multicolumn{2}{c}{0.7} & \multicolumn{2}{c}{0.4} & \multicolumn{2}{c}{0.1} \\
\hline Case & $\mathrm{N}$ & $\mathrm{P}$ & $\mathrm{N}$ & $\mathrm{P}$ & $\mathrm{N}$ & $\mathrm{P}$ & $\mathrm{N}$ & $\mathrm{P}$ \\
\hline Max. $F$ & 99.050 & 87.661 & 186.185 & 123.573 & 247.511 & 218.988 & 997.379 & 876.403 \\
Min. $F$ & 81.987 & 82.930 & 117.481 & 118.769 & 206.680 & 208.012 & 833.479 & 832.460 \\
Average $F$ & 86.544 & 83.814 & 123.801 & 119.474 & 215.292 & 210.070 & 847.479 & 845.779 \\
Std. Dev. & 3.941 & 1.194 & 9.073 & 1.305 & 6.723 & 3.304 & 35.955 & 16.568 \\
Final $F$ & 83.095 & 82.796 & 118.721 & 118.650 & 208.412 & 207.949 & 833.855 & 832.267 \\
\hline$t_{\text {ave }}$ & 10.95 & 25.22 & 7.71 & 19.36 & 7.80 & 12.87 & 5.83 & 10.18 \\
Converged & 76 & 86 & 72 & 83 & 68 & 79 & 61 & 72 \\
Solutions & & & & & & & &
\end{tabular}


It can be observed from Table 2 that none of the member length has extremely small value, and the stiffness matrix of the structure preserves regularity during optimization procedure. Note that no bound constraint for $q_{i}$ is active, however, the bounds for force densities are needed to prevent divergence of optimization process.

In order to obtain a distinct structural shape and topology, this best result is further optimized by solving problem (25) with volume constraint $\bar{V}=1\left(\mathrm{~m}^{3}\right)$ and $d_{i} \in[0.001,+\infty](\mathrm{m}), i=1,2, \ldots, \hat{m}$. The four nodes 7, 10, 11 and 12 are combined to a single node and members 4, 6, 13, 17, 18, 19, 22, 25 and 27, which reach the lower bound of member diameter, are removed before further optimization. The optimal solution of problem (25) is shown in Fig. 3(b), with the removal of members whose $d_{i}$ is equal to $d_{i, \text { lower, }}$ its compliance is slightly increased to $83.095 \mathrm{Nm}$. As we can see from Fig. 3, the procedure of further optimization "filters" the result and presents a more distinct solution.

Furthermore, we change the value of $\bar{V}$ to $0.7,0.4$ and $0.1\left(\mathrm{~m}^{3}\right)$, and find the optimal solutions as shown in Figs. 4, 5 and 6, respectively, and the compliances of optimal solutions of problem (25) are listed in Table 3. As seen from these figures, layouts of the optimal solutions corresponding to different values of $\bar{V}$ are similar, although they are not the same. The optimal solution for $\bar{V}=0.7$ $\left(\mathrm{m}^{3}\right)$ is asymmetric, which is similar to the case of optimal truss in Ref. [21]. Since the members of frame are rigidly jointed, the existence of collinear members connected to one node, which may cause singularity in stiffness of pin-jointed structure, is permitted. Thus, once the bounds of force density are carefully determined, the generation of extremely short members can be prevented keeping regularity of the stiffness matrix of the structure.

We further investigate the effectiveness of the proposed method of Case $\mathrm{P}$ with different values of $\bar{V}$. In this case, nodes 10 and 12, together with nodes 1, 2 and 3, are pin-supported for ensuring each of the member is in tension at self-equilibrium state to represent an auxiliary cable net, and no load is given for the cable net as the ground structure for finding the nodal location using the FDM. The loading condition of the frame to be optimized is the same as before.

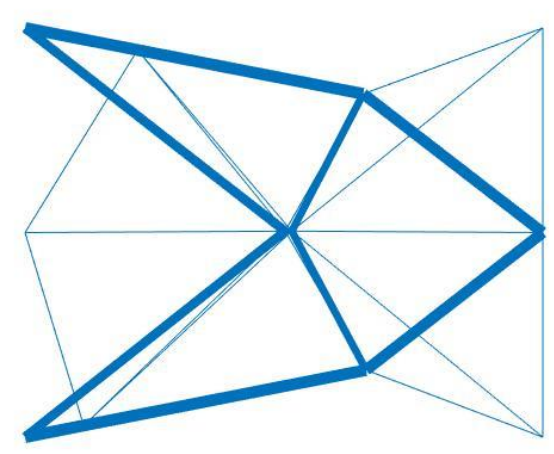

(a)

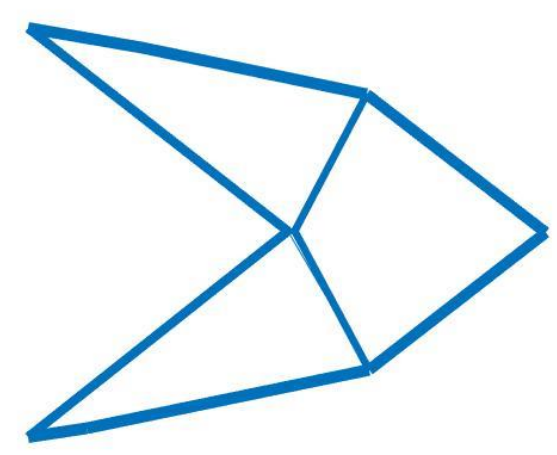

(b)

Fig. 7 Optimal shapes of Example 1 with Case $\mathrm{P}$ and $\bar{V}=1\left(\mathrm{~m}^{3}\right)$; (a) best result of problem (20), (b) optimal solution after solving problem (25)

Best result and optimal solution of problems (20) and (25) with $\bar{V}=1\left(\mathrm{~m}^{3}\right)$ are shown in Fig. 7, and the nodal locations of the best result are listed in Table 1, while the corresponding member diameters, force densities and member lengths are listed in Table 2. The values of $F$ after improvement are listed in Table 3, denoted as Final $F$, and their compliance values are close to those 
with the same values of $\bar{V}$, respectively. Note that the optimal shapes of Case P with other values of $\bar{V}$ are almost the same as Fig. 7 before further optimization, and similar to those in Figs. 4-6 after further optimization. Therefore, those figures are not shown here. It is seen from Fig. 7 that the optimal shape is defined using some members in the auxiliary cable net with supports in left and right boundaries.

Table 3 also shows the maximum, minimum, average and standard deviation of $F$ of problem (20) of 100 runs for each values of $\bar{V}$. It is seen from the table that the difference between max $F$ and $\min F$ with Case $\mathrm{N}$ is greater than that of Case $\mathrm{P}$, and the standard deviation also has larger value, indicating a wide range of variation in the solutions. This is mainly because if the force density is allowed to change from negative to positive real number or vice versa for Case $\mathrm{N}$, the free nodes move drastically and the possibility of convergence to a local optimal solution increases.

On the other hand, if all the members are allowed in tension only, the movement of free nodes become smooth due to invariant sign in force density and every node for Case $\mathrm{P}$ is balanced in tension. It is important to note for Case $\mathrm{P}$ that since only positive force density is allowed to exist, the boundary condition may be different from Case $\mathrm{N}$, however it has little influence on finding the optimal solution of problem (20) if the optimal shape can be successfully determined by a set of positive force densities. Some shapes during the optimization procedure are presented and illustrated in Appendix for a better interpretation of the proposed method with Case $\mathrm{N}$ and Case $\mathrm{P}$. The average computation time $t_{\text {ave }}$ of Example 1 among 100 random seeds are listed in Table 3, with a computer configuration of Intel Core i5 processor and 4GB RAM. As explained in Appendix, Case P has smoother convergence property than Case N; e.g., for $\bar{V}=1\left(\mathrm{~m}^{3}\right)$; therefore, nearly optimal solution can be easily obtained in Case P. However, the number of major iterations of SQP algorithm is 644 for Case P and 517 for Case N, and the Case P needs more computation time than the Case N, because the norm constraint for force density in problem (20) is to be satisfied and the feasible region in Case $\mathrm{P}$ is smaller than Case $\mathrm{N}$. Although the optimization procedure of Case $\mathrm{P}$ is less oscillatory, the step length can often be taken as a small value for satisfying the side constraints for $q_{i}$, requiring more iterations.

\subsection{Example 2}

In the second example, we investigate the optimal shape and topology of a 2-dimensional bridge frame with a $6 \times 1$ grid, where the 14 nodes are connected by 31 beam members, and the initial ground structure is shown in Fig. 8. The structure is pin-supported at node 1 and roller-supported at node 13. At each of the nodes 3, 5, 7, 9 and 11, a downward vertical load with unit magnitude $1 \mathrm{~N}$ is applied. Accordingly, these seven nodes are regarded as fixed nodes during the optimization procedure.

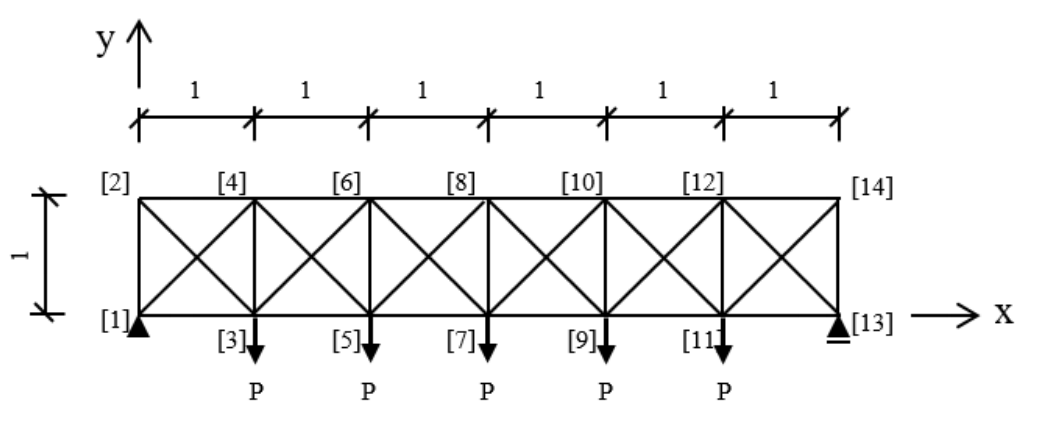


Fig. 8 Initial ground structure of Example 2

Again, $\bar{V}=1\left(\mathrm{~m}^{3}\right)$ is first assigned for this example. Because the summation of entries of each row in force density matrix $\boldsymbol{Q}$ is zero and $y$-coordinates of all fixed nodes are zero, one of the trivial solution for $\boldsymbol{y}_{\text {free }}$ is $\mathbf{0}$. Therefore, we assign upward reaction force $2.5 \mathrm{~N}$ at the supports 1 and 13 and include the $y$-directional equilibrium equations at the supports to be solved for $\boldsymbol{y}_{\text {free. }}$ The best result of problem (13) of Case $\mathrm{N}$ is presented in Fig. 9(a), with structural compliance $F=1221.03(\mathrm{Nm})$. Nodal locations are listed in Table 4, while the corresponding values of $\boldsymbol{q}, \boldsymbol{d}$, and $\boldsymbol{L}$ for all members are listed in Table 5. As seen from Fig. 9(a) and Table 5, the free nodes are separately located such that no extremely short member exists.

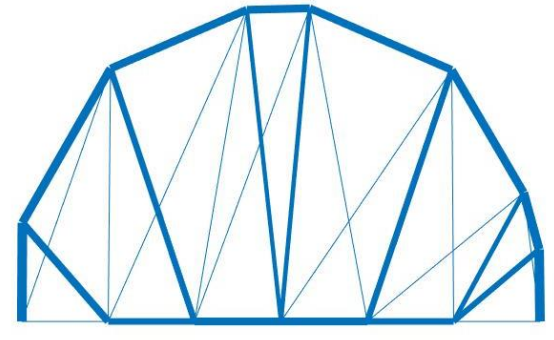

(a)

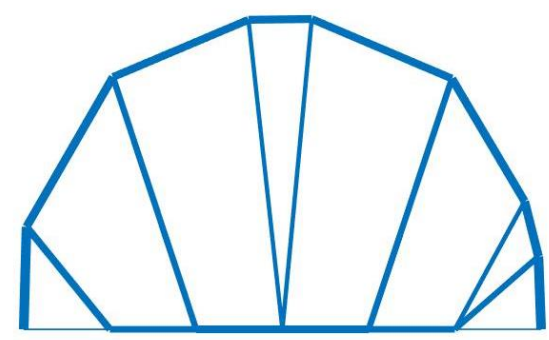

(b)

Fig. 9 Optimal shapes of Example 2 with Case $\mathrm{N}$ and $\bar{V}=1\left(\mathrm{~m}^{3}\right)$; (a) best result of problem (13), (b) optimal solution after solving problem (25)

Table 4. Nodal location of best result of Example 2 with $\bar{V}=1\left(\mathrm{~m}^{3}\right)$

\begin{tabular}{ccccc}
\hline \multirow{2}{*}{ Node } & \multicolumn{2}{c}{ Case N } & \multicolumn{2}{c}{ Case P } \\
\cline { 2 - 5 } & $x$ & $y$ & $x$ & $y$ \\
\hline 1 & 0 & 0 & 0 & 0 \\
2 & 0.0062 & 0.7462 & 0.0064 & 0.7583 \\
3 & 1 & 0 & 1 & 0 \\
4 & 1.0273 & 2.0677 & 0.7234 & 1.7475 \\
5 & 2 & 0 & 2 & 0 \\
6 & 2.6053 & 2.5714 & 1.3496 & 2.1902 \\
7 & 3 & 0 & 3 & 0 \\
8 & 3.3339 & 2.5842 & 3.0004 & 2.6822 \\
9 & 4 & 0 & 4 & 0 \\
10 & 4.978 & 2.0556 & 4.6505 & 2.1903 \\
11 & 5 & 0 & 5 & 0 \\
12 & 5.8098 & 1.0044 & 5.2764 & 1.7477 \\
13 & 6 & 0 & 6 & 0 \\
14 & 5.9893 & 0.5174 & 5.9936 & 0.7583 \\
15 & -- & -- & 0 & 3 \\
16 & -- & -- & 1 & 3 \\
17 & -- & -- & 2 & 3 \\
18 & -- & -- & 3 & 3 \\
19 & -- & -- & 4 & 3 \\
20 & -- & -- & 5 & 3 \\
21 & -- & -- & 6 & 3 \\
\hline & & & &
\end{tabular}


Table 5. Force density, diameter and length of best result of Example 2 with $\bar{V}=1\left(\mathrm{~m}^{3}\right)$

\begin{tabular}{|c|c|c|c|c|c|c|c|c|}
\hline \multirow{2}{*}{ Member } & \multirow{2}{*}{\multicolumn{2}{|c|}{ Nodes }} & \multicolumn{3}{|c|}{ Case N } & \multicolumn{3}{|c|}{ Case P } \\
\hline & & & $q_{i}$ & $d_{i}$ & $L_{i}$ & $q_{i}$ & $d_{i}$ & $L_{i}$ \\
\hline 1 & 1 & 2 & -2.08 & 0.304 & 0.846 & 0.381 & 0.302 & 0.858 \\
\hline 2 & 1 & 3 & 0.365 & 0.00431 & 1.000 & 0.122 & 0.00300 & 1.000 \\
\hline 3 & 1 & 4 & -0.343 & 0.00402 & 2.399 & 0.227 & 0.001 & 1.984 \\
\hline 4 & 2 & 3 & -0.398 & 0.238 & 1.305 & 0.00128 & 0.2341 & 1.312 \\
\hline 5 & 2 & 4 & -0.144 & 0.266 & 1.670 & 0.000790 & 0.264 & 1.221 \\
\hline 6 & 3 & 4 & 1.21 & 0.001 & 2.168 & 0.161 & 0.001 & 1.868 \\
\hline 7 & 3 & 5 & 0.706 & 0.207 & 1.000 & 0.102 & 0.203 & 1.000 \\
\hline 8 & 3 & 6 & -0.479 & 0.001 & 3.117 & 0.135 & 0.001 & 2.316 \\
\hline 9 & 4 & 5 & 0.0495 & 0.198 & 2.376 & 0.0227 & 0.145 & 2.245 \\
\hline 10 & 4 & 6 & -0.177 & 0.248 & 1.656 & 0.164 & 0.255 & 0.767 \\
\hline 11 & 5 & 6 & -0.276 & 0.001 & 2.739 & 0.000687 & 0.145 & 2.380 \\
\hline 12 & 5 & 7 & 0.111 & 0.244 & 1.000 & 0.203 & 0.244 & 1.000 \\
\hline 13 & 5 & 8 & 0.607 & 0.001 & 2.997 & 0.0164 & 0.001 & 2.956 \\
\hline 14 & 6 & 7 & -0.553 & 0.134 & 2.700 & 0.0135 & 0.001 & 2.823 \\
\hline 15 & 6 & 8 & 0.142 & 0.248 & 0.729 & 0.0231 & 0.247 & 1.722 \\
\hline 16 & 7 & 8 & 1.23 & 0.141 & 2.705 & 0.0318 & 0.187 & 2.782 \\
\hline 17 & 7 & 9 & 0.234 & 0.244 & 1.000 & 0.00720 & 0.244 & 1.000 \\
\hline 18 & 7 & 10 & -0.379 & 0.001 & 2.926 & 0.000117 & 0.001 & 2.823 \\
\hline 19 & 8 & 9 & 0.616 & 0.00424 & 2.766 & 0.00911 & 0.001 & 2.956 \\
\hline 20 & 8 & 10 & 0.224 & 0.247 & 1.727 & 0.000195 & 0.247 & 1.721 \\
\hline 21 & 9 & 10 & -0.477 & 0.196 & 2.367 & 0.00513 & 0.145 & 2.381 \\
\hline 22 & 9 & 11 & 0.495 & 0.206 & 1.000 & 0.0991 & 0.203 & 1.000 \\
\hline 23 & 9 & 12 & 0.340 & 0.0116 & 2.120 & 0.0759 & 0.145 & 2.245 \\
\hline 24 & 10 & 11 & 0.494 & 0.001 & 2.156 & 0.165 & 0.001 & 2.317 \\
\hline 25 & 10 & 12 & -0.181 & 0.261 & 1.340 & 0.219 & 0.255 & 0.767 \\
\hline 26 & 11 & 12 & -0.224 & 0.152 & 1.369 & 0.0347 & 0.001 & 1.868 \\
\hline 27 & 11 & 13 & 0.396 & 0.00409 & 1.000 & 0.0940 & 0.003 & 1.000 \\
\hline 28 & 11 & 14 & 0.295 & 0.183 & 1.166 & 0.00310 & 0.234 & 1.313 \\
\hline 29 & 12 & 13 & -2.06 & 0.001 & 1.121 & 0.234 & 0.001 & 1.984 \\
\hline 30 & 12 & 14 & -0.0650 & 0.283 & 0.519 & 0.000239 & 0.264 & 1.222 \\
\hline 31 & 13 & 14 & -0.363 & 0.303 & 0.618 & 0.433 & 0.302 & 0.858 \\
\hline 32 & 2 & 15 & -- & -- & -- & 0.144 & 0.001 & 2.241 \\
\hline 33 & 2 & 16 & -- & -- & -- & 0.00156 & 0.001 & 2.452 \\
\hline 34 & 15 & 4 & -- & -- & -- & 0.270 & 0.001 & 1.446 \\
\hline 35 & 15 & 16 & -- & -- & -- & 0.0706 & 0.001 & 1.000 \\
\hline 36 & 4 & 16 & -- & -- & -- & 0.171 & 0.001 & 1.282 \\
\hline 37 & 4 & 17 & -- & -- & -- & 0.107 & 0.001 & 1.788 \\
\hline 38 & 16 & 6 & -- & -- & -- & 0.284 & 0.001 & 0.882 \\
\hline 39 & 16 & 17 & -- & -- & -- & 0.139 & 0.001 & 1.000 \\
\hline 40 & 6 & 17 & -- & -- & -- & 0.163 & 0.001 & 1.038 \\
\hline 41 & 6 & 18 & -- & -- & -- & 0.0498 & 0.001 & 1.838 \\
\hline
\end{tabular}




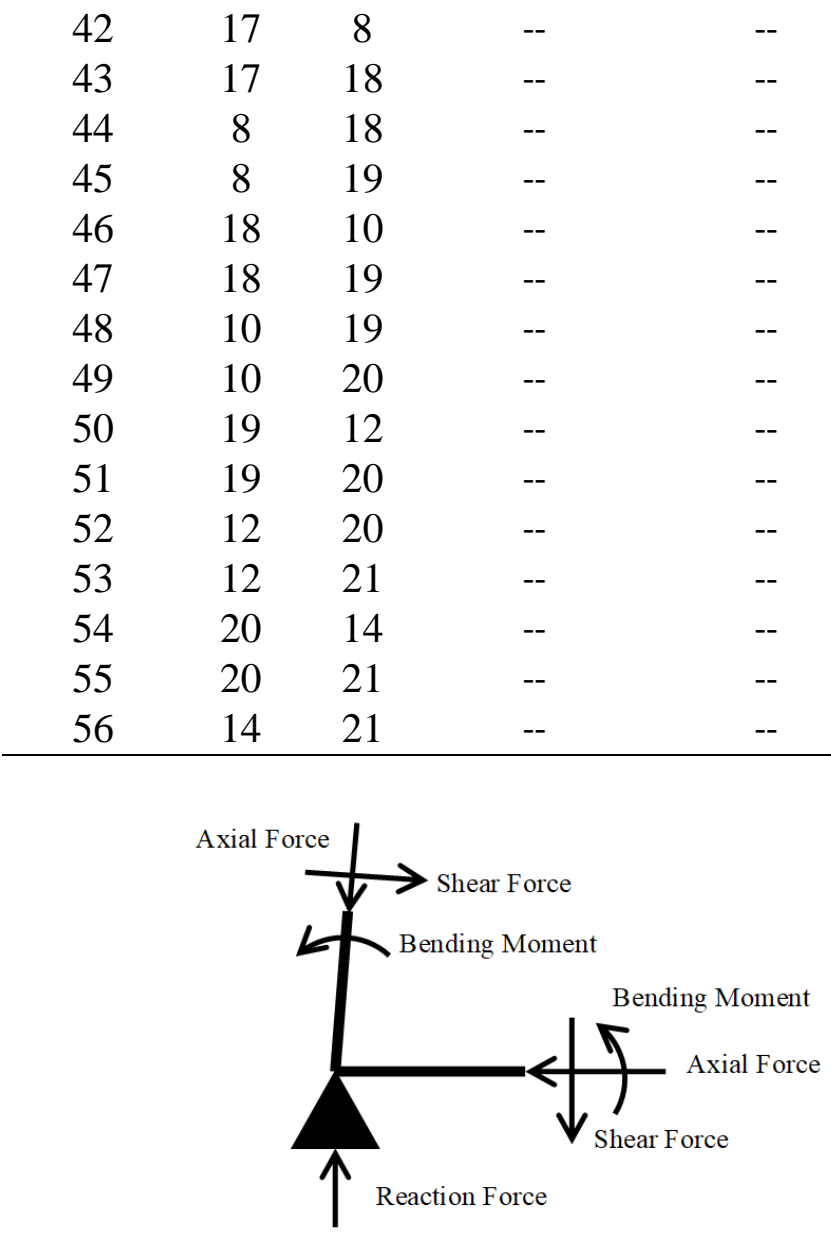

(a)

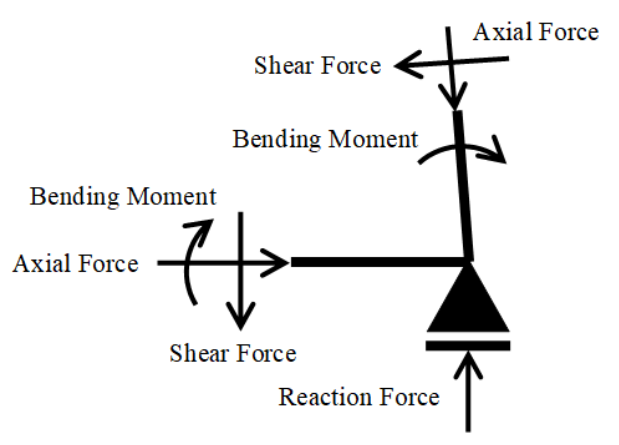

(b)

Fig. 10 Illustration of equilibrium condition; (a) pin-supported node 1 and (b) roller-supported node 31

The optimal solution is obtained by solving problem (25) with $\bar{V}=1\left(\mathrm{~m}^{3}\right)$ as shown in Fig. $9(\mathrm{~b})$. After removal of thin members with $d_{i, \text { lower }}=0.001(\mathrm{~m})$, the compliance $F$ is reduced to $1219.22 \mathrm{Nm}$. It should be noted that members 2 and 27 connecting nodes $(1,3)$ and nodes $(11,13)$, respectively, do not vanish in Fig. 9(b), making the structure globally stable. This property is different from the optimal shape of pin-jointed truss [14], where members 2 and 27 do not exist. This is because only axial force equilibrium condition has to be satisfied for truss structure; however, for rigidly-jointed frame structure, axial force, shear force and bending moment simultaneously exist in the member, leading to the slight inclination of members 1 and 31, as shown in Fig. 10. Therefore, forces in members 2 and 27 are not zero-force member and are necessary for satisfying the equilibrium conditions at supports. 


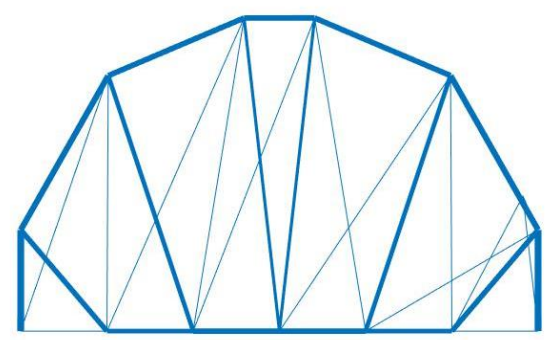

(a)

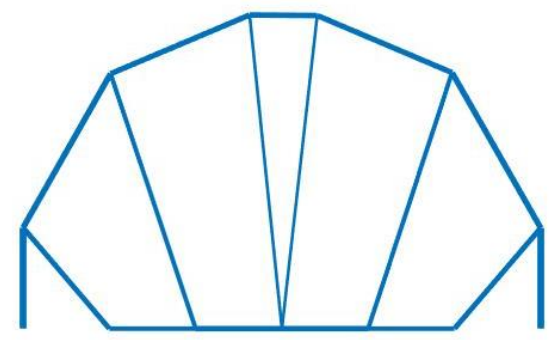

(b)

Fig. 11 Optimal shapes of Example 2 with Case $\mathrm{N}$ and $\bar{V}=0.1\left(\mathrm{~m}^{3}\right)$; (a) best result of problem (13), (b) optimal solution after solving problem (25)

Table 6. Statistical result, final values of compliance $F(\mathrm{Nm})$ and CPU time (sec) of 100 results of Example 2 with different $\bar{V}\left(\mathrm{~m}^{3}\right)$

\begin{tabular}{ccccc}
\hline $\bar{V}\left(\mathrm{~m}^{3}\right)$ & \multicolumn{2}{c}{1} & \multicolumn{2}{c}{0.1} \\
\hline Case & $\mathrm{N}$ & $\mathrm{P}$ & $\mathrm{N}$ & $\mathrm{P}$ \\
\hline Max $F$ & 7898.12 & 1276.47 & 47039.81 & 13405.61 \\
Min $F$ & 1221.03 & 1217.43 & 12255.81 & 12232.50 \\
Average $F$ & 2252.89 & 1232.22 & 15520.27 & 12448.70 \\
Std. Dev. & 1912.16 & 21.2445 & 6842.66 & 310.84 \\
Final $F$ & 1219.22 & 1217.07 & 12255.04 & 12230.92 \\
\hline$t_{\text {ave }}$ & 22.65 & 25.99 & 12.47 & 16.01 \\
Converged & 85 & 97 & 82 & 93 \\
Solutions & & & & \\
\hline
\end{tabular}

The same optimization procedure is carried out with $\bar{V}=0.1\left(\mathrm{~m}^{3}\right)$, and compliance of the improved optimal solution is listed in Table 6. It can be also found from Fig. 11 that no melting nodes exist for this case. Although the frame in Fig. 11 has only six upper nodes, members 25 and 30 connecting nodes $(10,12)$ and $(12,14)$, respectively, are colinear, and node 12 exists on the member connecting nodes $(10,14)$. Furthermore, with the decrease of upper bound volume, the diameters of members 2 and 27 are closer to $d_{i, \text { lower }}$ and these members are removed. However, the best optimal result is stable owing to small bending stiffness in members 1 and 31 .

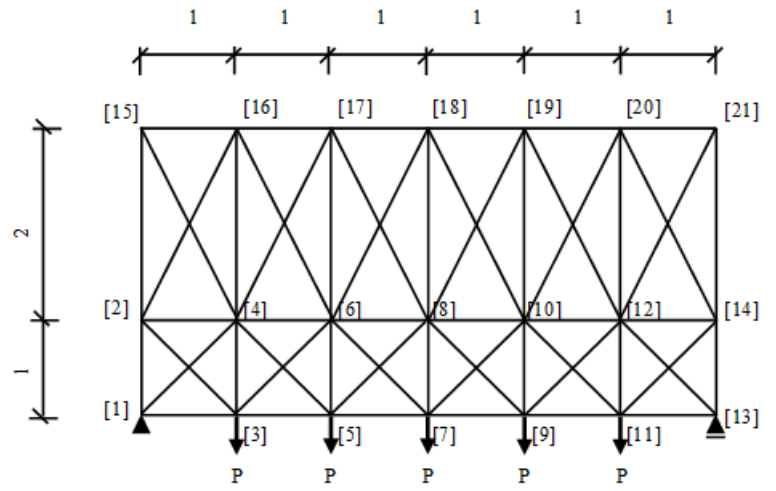

Fig. 12 Initial ground structure for Case P of Example 2 


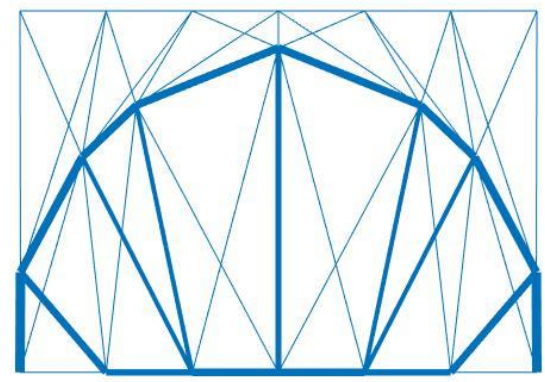

(a)

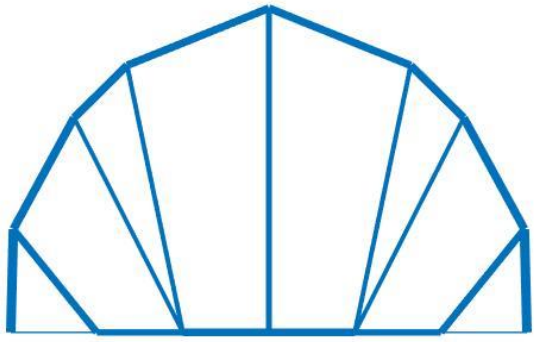

(b)

Fig. 13 Optimal shapes of Example 2 with Case P and $\bar{V}=1\left(\mathrm{~m}^{3}\right)$; (a) best result of problem (20), (b) optimal solution after solving problem (25)

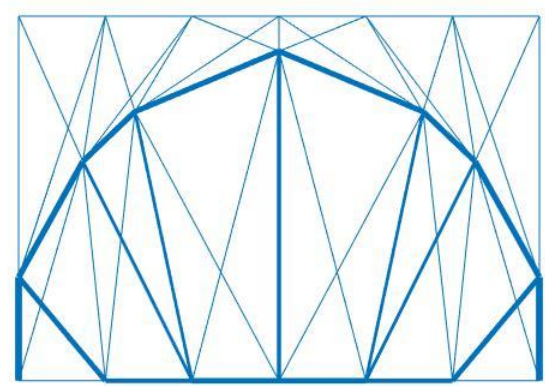

(a)

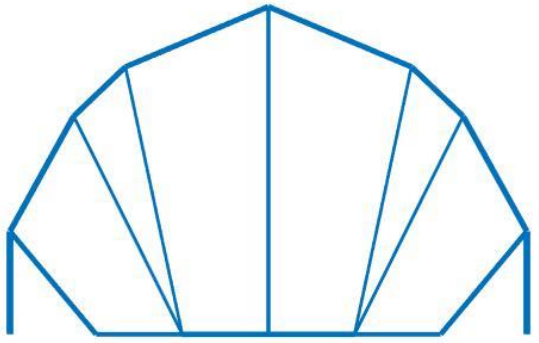

(b)

Fig. 14 Optimal shapes of Example 2 with Case P and $\bar{V}=0.1\left(\mathrm{~m}^{3}\right)$; (a) best result of problem (20), (b) optimal solution after solving problem (25)

Optimization problems (20) and (25) for Example 2 are solved also with Case P. Seven additional nodes 15-21 exist at the top of initial structure, as shown in Fig. 12. By fixing the locations of nodes $1,3,5,7,9,11,13,15-21$, we have an auxiliary cable net to define the nodal locations. In order to allow the free nodes to move in a wide range, the $y$-coordinates of the seven additional nodes have a large value $3 \mathrm{~m}$. This way, a kind of cable net is generated for solving Eq. (10) to obtain $\boldsymbol{x}_{\text {free }}$ and $y_{\text {free. }}$

The compliance values of improved optimal solutions are listed in Table 6, and Figs. 13 and 14 show the optimal solutions for $\bar{V}=1$ and $0.1\left(\mathrm{~m}^{3}\right)$, respectively. Although the final structural layout of these four solutions are symmetric with respect to the middle vertical axis, symmetry constraints are not enforced explicitly. Through observation from Table 6 , one can realize that values of $F$ of improved optimal solution with the same $\bar{V}$ are very close in both cases. It is worth noting that the initial ground structures for Case $\mathrm{N}$ and Case $\mathrm{P}$ are different; however, the purpose of adding nodes and members is merely to obtain a self-equilibrium state of free nodes with tension state, and none of the additional nodes are allowed to move. Therefore, the optimal shapes of both cases are still controlled by the same free nodes, i.e., nodes 2, 4, 6, 8 and 10, and similar optimal solutions can be found by the proposed method if the coordinates of free nodes of optimal shape can be determined in their feasible domain of force density.

Table 6 also lists the statistical result of 100 runs, together with the computation time $t_{\text {ave }}$ with the same computer configuration as Example 1 and the number of converged solutions within 100 different trials. It can be observed that the value of maximum $F$ among 100 runs can be 5 or 6 times 
the value of minimum $F$ for Case $\mathrm{N}$, indicating the tendency of being trapped in a local optimal solution or even diverge to some extent, and its standard deviation is also greater than that of Case P. However, the values of average $F$ for both cases are within an acceptable range, about $20 \%$ larger than the minimum $F$.

\subsection{Example 3}

The third example is optimization of a grid-like shear wall subjected to a horizontal force of $1 \mathrm{~N}$ at the top as shown in Fig. 15. The structure is pin-supported at nodes 1 and 31, and because the border of shear wall is usually required to keep rectangle after optimization, nodes on the border frame, i.e., $2,3,4,5,6,10,11,15,16,20,21,25,26,30,32,33,34$ and 35, as well as nodes 1 and 31, are classified as fixed nodes, and the remaining 15 nodes are the free nodes. Members of border and inner lattice are depicted by thick and thin black lines in Fig. 15, respectively. The cross-section diameters of border frame members are fixed at $0.1 \mathrm{~m}$ and remain unchanged during the optimization procedure. Design variables are the cross-section diameters and force densities of all the inner lattice members.

In this example, only Case $\mathrm{P}$ is considered, and Fig. 16 shows the optimization results by solving problems (20) and (25) with $\bar{V}=1$.

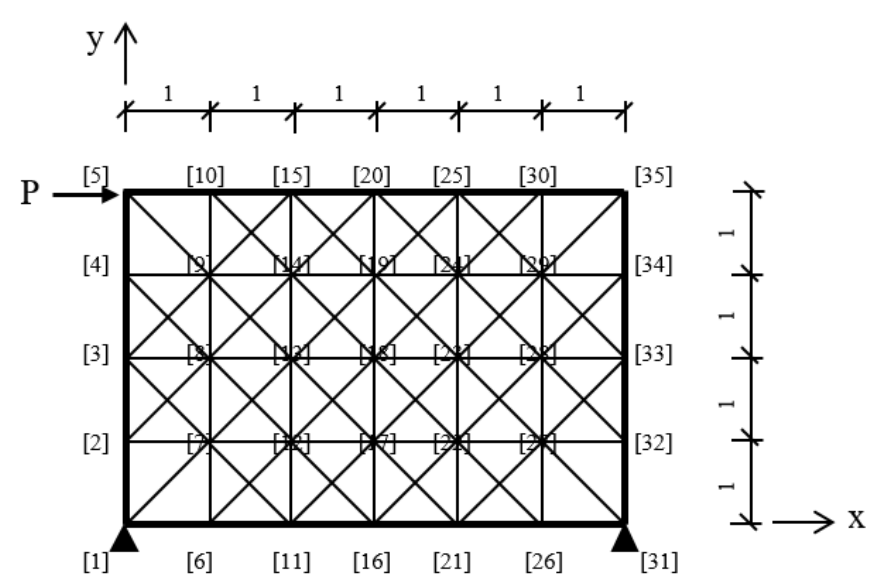

Fig. 15 Initial ground structure of Example 3

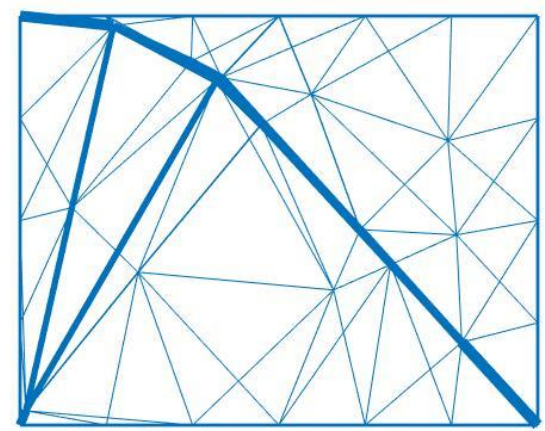

(a)

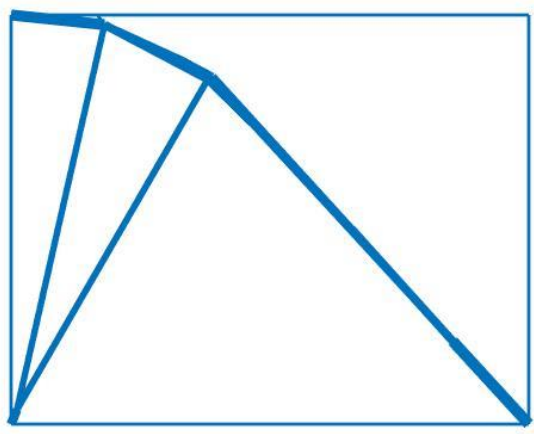

(b)

Fig. 16 Optimal shapes of Example 3 with Case $\mathrm{P}$ and $\bar{V}=1\left(\mathrm{~m}^{3}\right)$; (a) best result of problem (20), (b) optimal solution after solving problem (25)

It can be observed from Fig. 16(a) that no extremely short member exists, although some of the 
nodes are closely spaced and connected mainly due to the low axial force in the self-equilibrium state, and the diameters of many members reach $d_{i, \text { lower }}$ Then, the final optimal solution is obtained as shown in Fig. 16(b) by further solving problem (25), where the compliance value is $103.0494 \mathrm{Nm}$. Next, problems (20) and (25) are solved with the change of upper volume bound $\bar{V}$ to $0.7,0.4$ and $0.1\left(\mathrm{~m}^{3}\right)$. The optimal solutions are shown in Figs. 17-19, respectively, and the corresponding compliances after improvement are listed in Table 7. For $\bar{V}=1$ and $0.7\left(\mathrm{~m}^{3}\right)$, the optimal solutions are similar, however, the shape and topology of optimal solution becomes different when $\bar{V}$ decrease to 0.4 and $0.1\left(\mathrm{~m}^{3}\right)$. This is mainly because long members cannot exist if the diameter becomes small as $\bar{V}$ is decreased.

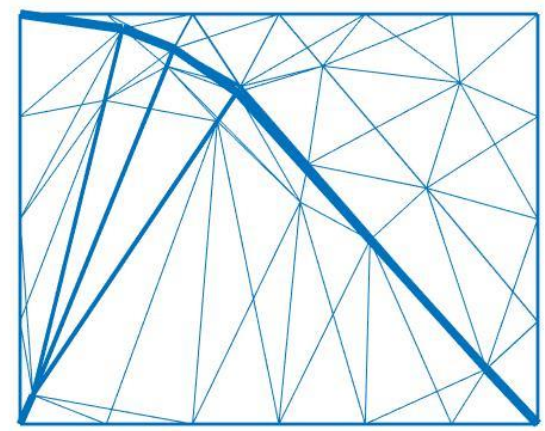

(a)

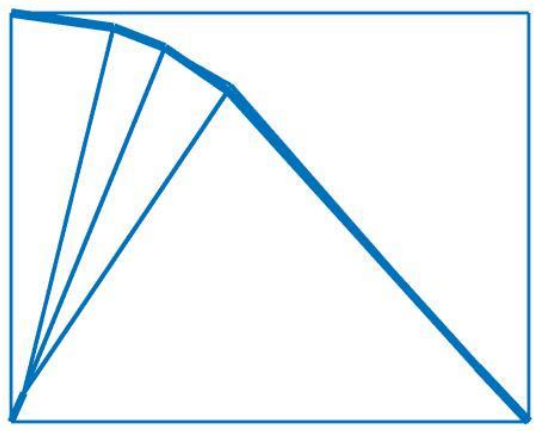

(b)

Fig. 17 Optimal shapes of Example 3 with Case $\mathrm{P}$ and $\bar{V}=0.7\left(\mathrm{~m}^{3}\right)$; (a) best result of problem (20), (b) optimal solution after solving problem (25)

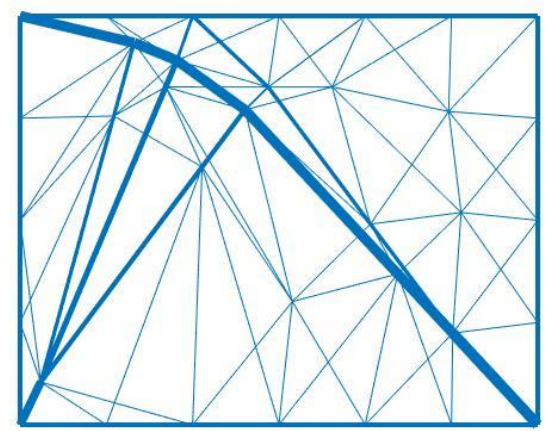

(a)

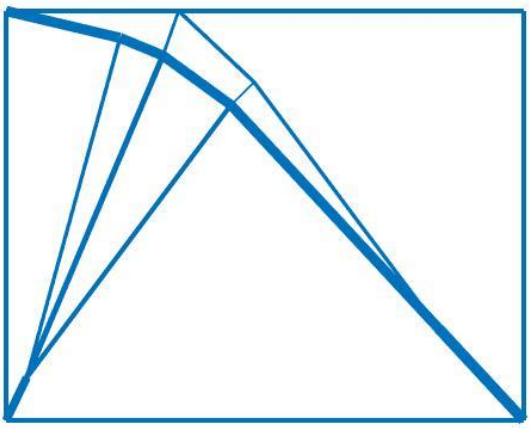

(b)

Fig. 18 Optimal shapes of Example 1 with Case P and $\bar{V}=0.4\left(\mathrm{~m}^{3}\right)$; (a) best result of problem (20), (b) optimal solution after solving problem (25) 


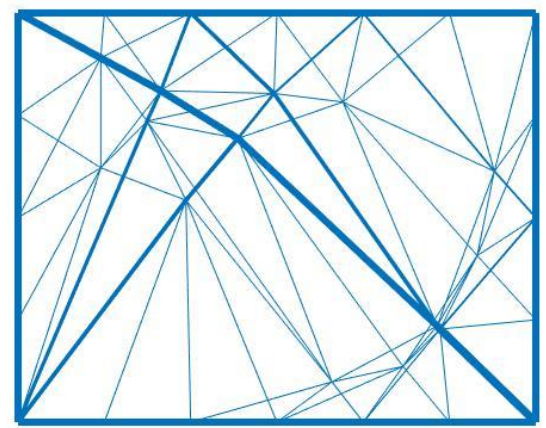

(a)

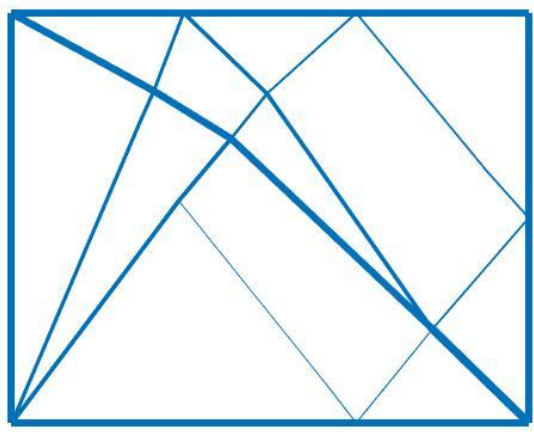

(b)

Fig. 19 Optimal shapes of Example 3 with Case P and $\bar{V}=0.1\left(\mathrm{~m}^{3}\right)$; (a) best result of problem (20), (b) optimal solution after solving problem (25)

Table 7. Statistical result, initial and final values of compliance $F(\mathrm{Nm})$ and CPU time (sec) of 100 results of Example 3 with different $\bar{V}\left(\mathrm{~m}^{3}\right)$

\begin{tabular}{ccccc}
\hline $\bar{V}\left(\mathrm{~m}^{3}\right)$ & 1 & 0.7 & 0.4 & 0.1 \\
\hline Max $F$ & 107.595 & 146.811 & 245.683 & 889.966 \\
Min $F$ & 103.829 & 144.110 & 242.160 & 840.453 \\
Average $F$ & 105.315 & 145.700 & 244.180 & 851.771 \\
Std. Dev & 0.9778 & 0.4579 & 0.6406 & 10.814 \\
Final $F$ & 103.050 & 143.794 & 242.142 & 840.231 \\
Initial $F$ & 388.792 & 465.937 & 626.432 & 1517.843 \\
\hline$t_{\text {ave }}$ & 27.45 & 27.44 & 22.30 & 21.47 \\
\hline
\end{tabular}

The maximum, minimum, average and standard deviation of $F$ among 100 random seeds, as well as $t_{\text {ave, }}$, are shown in Table 7. Compliance of initial ground structure where the inner lattice members are evenly distributed is also calculated and listed in Table 7 as Initial $F$ for comparison to the optimal solution with the same $\bar{V}$. It can be seen from Table 7 that the compliance is significantly decreased through optimization by the proposed method.

\section{Conclusion}

A new method has been presented for geometry and topology optimization of plane frame structure using force density for defining the nodal locations of free nodes. An auxiliary truss or cable net with different boundary and loading conditions is used for defining the nodal locations.

In order to prevent the generation of melting nodes in the structure, the side constraint on member length is indirectly assigned by limiting the force density value of each member. Two cases of lower bound of force density are given; namely, Case $\mathrm{N}$ for negative lower bound and Case $\mathrm{P}$ for small positive lower bound, which correspond to truss and cable net, respectively, for the definition of auxiliary structure for defining nodal coordinates. Sensitivity coefficients of objective and constraint functions with respect to force density and member diameter are also derived to accelerate the optimization procedure. After obtaining the optimization results, the geometry and topology of the structure are further improved in order to achieve a distinct final solution, with no extremely thin member or closely space nodes. 
Three numerical examples are presented to illustrate the effectiveness of the proposed method. It has been shown that reasonable shape and topology can be successfully found for both Case $\mathrm{N}$ and Case P. However, comparison of results obtained from 100 different trials shows that Case $\mathrm{P}$ is more stable, i.e., has smaller standard deviation, than Case N. An approximate optimal solution can be obtained smoothly by assigning a positive lower bound for force density, although convergence to a strict optimal solution is slow.

It is interesting to note in the example of bridge frame that a thin horizontal member is needed at the roller support to be at equilibrium with the shear force of the thick vertical member, although such member is not needed for a truss or a frame with thin vertical member corresponding to a small upper bound of structural volume. Furthermore, the coordinates of free nodes cannot be determined using FDM, if the vertical coordinates of fixed nodes have the same value. In this case, an equilibrium condition with the reaction force at a support is included to successfully obtain the nodal coordinates of free nodes.

\section{Acknowledgements}

This research is partially supported by China Scholarship Council (File No. 201806050114).

\section{Appendix}

The intermediate solutions in optimization procedure of Example 1 with $\bar{V}=1$ are shown in Figs. $\mathrm{A} 1$ and $\mathrm{A} 2$ for Cases $\mathrm{P}$ and $\mathrm{N}$, respectively. Note that both the results correspond to the best results of problem (20), and the red and blue color of members stand for positive and negative member forces, respectively. The figure at the top-right of Fig. A2 is trimmed to be consistent with the others, although some of the nodes and members are cut off.

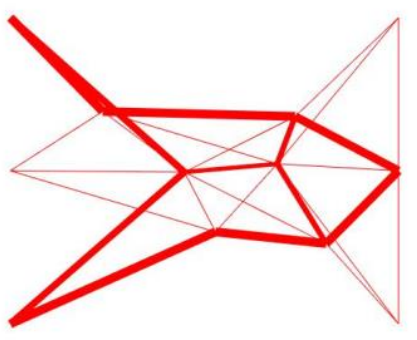

Step 1

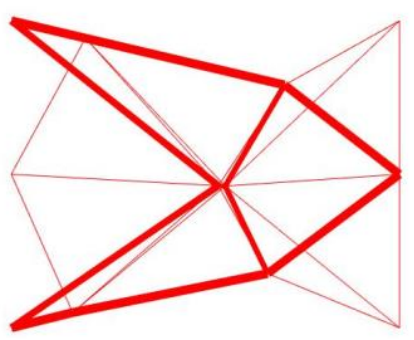

Step 360

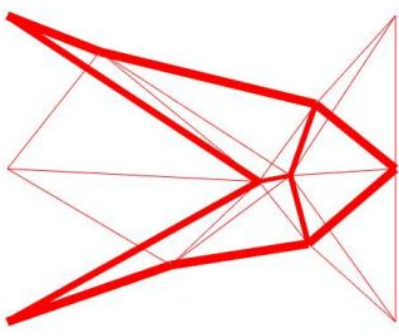

Step 120

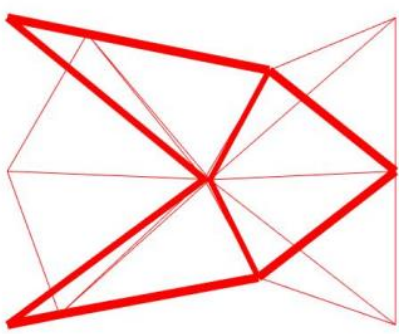

Step 480

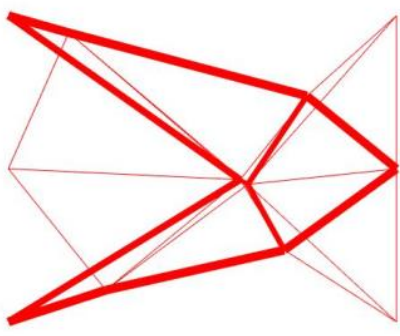

Step 240

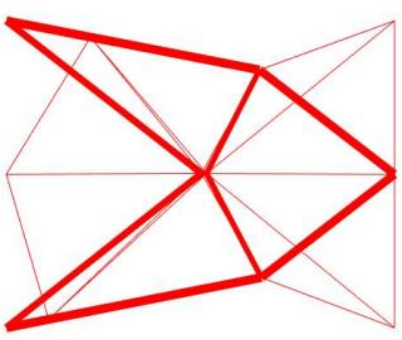

Step 600

Fig. A1 Intermediate solutions of Example 1 with Case P 


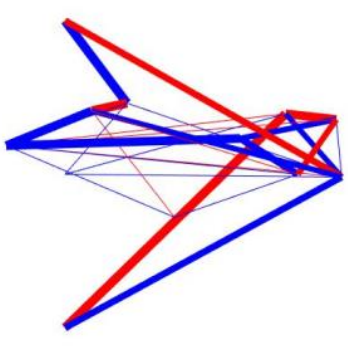

Step 1

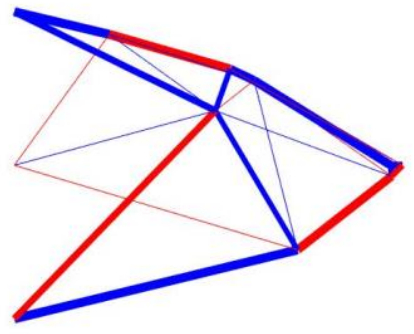

Step 300

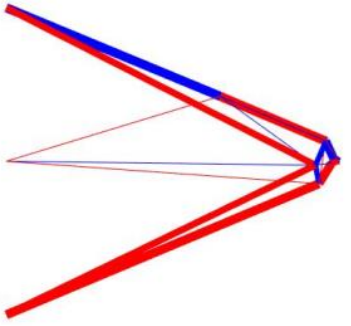

Step 100

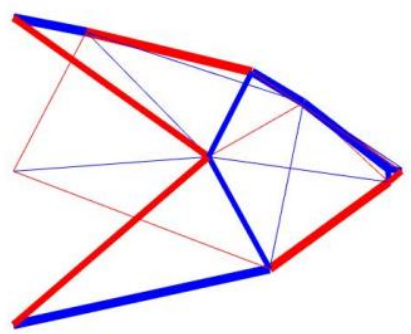

Step 400

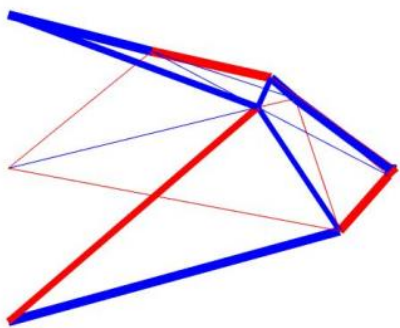

Step 200

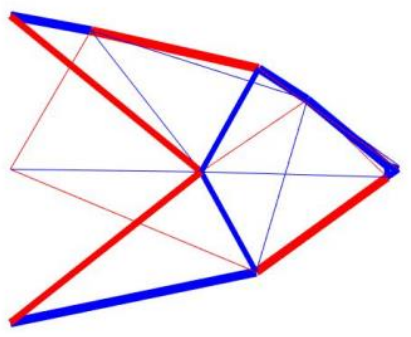

Step 500

Fig. A2 Intermediate solutions of Example 1 with Case N

As seen from Figs. A1 and A2, in Case $\mathrm{N}$ the structure undergoes drastic variation at the beginning mainly due to the change of signs of the force; on the other hand, iteration in optimization procedure with Case P has "smoother" shape variation leading to a monotonic convergence to the solution.

\section{Reference}

1. Maxwell JC (1870) On Reciprocal Figures, Frames, and Diagrams of Forces. Trans R Soc Edinburgh 161-207. https://doi.org/10.1017/S0080456800026351

2. Michell AGM (1904) The Limits of Economy of lllaterial in Frame-structures . Philos Mag Ser 6 8:589-597. https://doi.org/10.1080/14786440409463229

3. M.P.Bendose, A.Ben-Tal, J.Zowe (1994) Optimization methods for truss geometry and topology design. Structrual Optim 7:141-159. https://doi.org/10.1117/12.660870

4. Stolpe M (2016) Truss optimization with discrete design variables: a critical review. Struct Multidiscip Optim 53:349-374. https://doi.org/10.1007/s00158-015-1333-x

5. Bendsøe MP, Sigmund O (2003) Topology optimization: theory, methods, and applications. Springer

6. Ohsaki M (2010) Optimization of Finite Dimensional Structures. CRC Press

7. Lewiński T, Sokół T, Graczykowski C (2019) Michell structures. Springer International Publishing

8. Kirsch U, Taye S (1986) On optimal topology of grillage structures. Eng Comput 1:229- 


\section{3. https://doi.org/10.1007/BF01200139}

9. Adil B, Cengiz B (2019) Optimal design of truss structures using weighted superposition attraction algorithm. Eng Comput. https://doi.org/10.1007/s00366-019-00744-X

10. Dorn WS, Gomory RE, Greenberg HJ (1964) Automatic design of optimal structures. J Mech 3:25-52

11. Topping BH V. (1983) Shape Optimization of Skeletal Structures: A Review. J Struct Eng 109:1933-1951. https://doi.org/10.1061/(asce)0733-9445(1983)109:8(1933)

12. Gil L, Andreu A (2001) Shape and cross-section optimization of a truss structure. Comput Struct 79:681-689. https://doi.org/10.1016/S0045-7949(00)00182-6

13. Lamberti L, Pappalettere C (2003) A numerical code for lay-out optimization of skeletal structures with sequential linear programming. Eng Comput 19:101-129. https://doi.org/10.1007/s00366-003-0258-y

14. Achtziger W (2007) On simultaneous optimization of truss geometry and topology. Struct Multidiscip Optim 33:285-304. https://doi.org/10.1007/s00158-006-0092-0

15. Ohsaki M (1998) Simultaneous optimization of topology and geometry of a regular plane truss. Comput Struct 66:69-77. https://doi.org/10.1016/S0045-7949(97)00050-3

16. Guo Xu Liu Wei Simultaneous shape and topology optimization of truss under local and global stability constraints

17. Czarnecki S (2003) Compliance optimization of the truss structures. Comput. Assist. Mech. Eng. Sci. 10:117-137

18. Sokół T (2011) A 99 line code for discretized Michell truss optimization written in Mathematica. Struct Multidiscip Optim 43:181-190. https://doi.org/10.1007/s00158-010$0557-\mathrm{Z}$

19. Schek HJ (1974) The force density method for form finding and computation of general networks. Comput Methods Appl Mech Eng 3:115-134. https://doi.org/10.1016/00457825(74)90045-0

20. Zhang JY, Ohsaki M (2006) Adaptive force density method for form-finding problem of tensegrity structures. Int J Solids Struct 43:5658-5673

21. Ohsaki M, Hayashi K (2017) Force density method for simultaneous optimization of geometry and topology of trusses. Struct Multidiscip Optim 56:1157-1168. https://doi.org/10.1007/s00158-017-1710-8

22. Kimura T, Ohsaki M, Yamaoka Y Shape and topology optimization of latticed shear wall utilising contact to existing frame. J Struct Constr Eng (Transactions AIJ) 84:385-391. (in Japanese) 
23. Descamps B, Filomeno Coelho R (2014) The nominal force method for truss geometry and topology optimization incorporating stability considerations. Int J Solids Struct 51:23902399. https://doi.org/10.1016/j.ijsolstr.2014.03.003

24. Zienkiewicz OC, Taylor RL, Zhu JZ (2005) The Finite Element Method: Its Basis and Fundamentals (sixth ed.). Elsevier, Oxford

25. Zhang JY, Ohsaki M (2015) Tensegrity Structures: Form, Stability, and Symmetry. Springer

26. Kanno Y, Ohsaki M (2003) Minimum principle of complementary energy of cable networks by using second-order cone programming. Int J Solids Struct 40:4437-4460.

https://doi.org/10.1016/S0020-7683(03)00215-4

27. Kirsch U, Bogomolni M, Sheinman I (2007) Efficient structural optimization using reanalysis and sensitivity reanalysis. Eng Comput 23:229-239.

https://doi.org/10.1007/s00366-007-0062-1

28. Ohsaki M, Ikeda K (2010) Stability and Optimization of Structures: Generalized Sensitivity Analysis. Springer, Sendai

29. Arora JS, Haug EJ (1979) Methods of Design Sensitivity Analysis in Structural Optimization. AIAA J 17:970-974. https://doi.org/10.2514/3.61260

30. Achtziger W (1998) Multiple-load truss topology and sizing optimization: Some properties of minimax compliance. J Optim Theory Appl 98:255-280.

https://doi.org/10.1023/A:1022637216104

31. Mathworks (2018) Optimzation Toolbox User's Guide R2018a 